\title{
IRF3 promotes adipose inflammation and insulin resistance and represses browning
}

\author{
Manju Kumari, ${ }^{1}$ Xun Wang, ${ }^{1}$ Louise Lantier, ${ }^{2}$ Anna Lyubetskaya, ${ }^{1}$ Jun Eguchi, ${ }^{3}$ Sona Kang, ${ }^{1}$ Danielle Tenen, ${ }^{1}$ Hyun Cheol Roh, ${ }^{1}$ \\ Xingxing Kong, 'Lawrence Kazak, ${ }^{4}$ Rasheed Ahmad, ${ }^{5}$ and Evan D. Rosen ${ }^{1}$ \\ 'Division of Endocrinology, Beth Israel Deaconess Medical Center, Boston, Massachusetts, USA. ²Department of Molecular Physiology and Biophysics, Vanderbilt University, Nashville, Tennessee, USA. \\ ${ }^{3}$ Department of Nephrology, Rheumatology, Endocrinology and Metabolism, Okayama University Graduate School of Medicine, Dentistry and Pharmaceutical Sciences, Okayama, Japan. ${ }^{4}$ Dana-Farber Cancer \\ Institute and Department of Cell Biology, Harvard Medical School, Boston, Massachusetts, USA. Immunology and Innovative Cell Therapy Unit, Dasman Diabetes Institute, Kuwait City, Kuwait.
}

\begin{abstract}
The chronic inflammatory state that accompanies obesity is a major contributor to insulin resistance and other dysfunctional adaptations in adipose tissue. Cellular and secreted factors promote the inflammatory milieu of obesity, but the transcriptional pathways that drive these processes are not well described. Although the canonical inflammatory transcription factor NF- $\mathrm{KB}$ is considered to be the major driver of adipocyte inflammation, members of the interferon regulatory factor (IRF) family may also play a role in this process. Here, we determined that IRF3 expression is upregulated in the adipocytes of obese mice and humans. Signaling through TLR3 and TLR4, which lie upstream of IRF3, induced insulin resistance in murine adipocytes, while IRF3 knockdown prevented insulin resistance. Furthermore, improved insulin sensitivity in IRF3-deficient mice was associated with reductions in intra-adipose and systemic inflammation in the high fat-fed state, enhanced browning of subcutaneous fat, and increased adipose expression of CLUT4. Taken together, the data indicate that IRF3 is a major transcriptional regulator of adipose inflammation and is involved in maintaining systemic glucose and energy homeostasis.
\end{abstract}

\section{Introduction}

One of the major concepts emerging from the last 2 decades of metabolism research is the notion of "metainflammation," the state of chronic low-grade inflammation that develops during periods of overnutrition $(1,2)$. This manifests itself most notably through significant alterations in the number and composition of the immune cell populations that reside in adipose tissue, including a dramatic increase in the ratio of classically activated M1 macrophages versus alternatively activated M2 macrophages $(3,4)$. The result is a proinflammatory cytokine milieu that is believed to promote insulin resistance in surrounding adipocytes. While it is still unclear how overnutrition is sensed by the adipocyte, a role for pattern recognition receptors has been proposed, including members of the Toll-like receptor (TLR) family (5). TLRs respond to various pathogen-associated molecules that include fatty acids, endotoxin, unmethylated DNA, and double-stranded RNA; ligation by such agents triggers a signal transduction cascade that differs for different TLRs but which inevitably results in the transcription of various chemokines and cytokines. While TLR signaling has been classically described in cells of the innate immune system, adipocytes themselves express several TLRs and the relevant downstream adaptors and signal transduction elements $(5,6)$. Furthermore, activation of adipocyte TLR4 in particular has been shown to promote insulin resistance, such that mice lacking TLR4 are protected from many of the adverse metabolic consequences of overfeeding, including insulin resistance (7-9).

Conflict of interest: E.D. Rosen receives research funding from Merck.

Submitted: December 16, 2015; Accepted: May 12, 2016.

Reference information: J Clin Invest. 2016;126(8):2839-2854. doi:10.1172/JCI86080.
Signaling by TLRs, including TLR4, converges on a small set of transcription factors that are believed to mediate the transcriptional response promoting inflammation. Most prominent among these factors is NF- $\kappa \mathrm{B}$, which is normally sequestered in the cytosol bound to the inhibitor IאB protein (10). Upon phosphorylation by IKK- $\beta$, IкB is degraded, allowing NF- $\kappa$ B to translocate to the nucleus and bind to its target genes. On the basis of inference from studies in immune cells, NF- $\mathrm{kB}$ has been supposed to be the major transcriptional driver of inflammation in the adipocyte, although this has never been directly proven, and is in fact controverted by several studies (11-15). We thus raise the question: how are proinflammatory signals transduced into altered gene expression of inflammatory markers, and which transcription factors mediate the enhanced inflammatory state of adipose tissue in the setting of obesity?

Another class of transcription factors that is intimately involved in both innate and adaptive immunity is the interferon regulatory factor (IRF) family, comprising 9 members (IRF1IRF9). IRFs have been implicated in virtually every aspect of immune function, ranging from macrophage development and function to $\mathrm{T}$ and $\mathrm{B}$ cell lymphopoiesis and antiviral defense, among others (16). We have previously identified a role for several IRFs in adipogenesis, and have further implicated IRF4 as a key determinant of adipocyte lipid handling (in the context of fasting and feeding) and of thermogenesis (in the context of cold exposure) (17-19). Several other IRFs are expressed in adipocytes, and may be activated by TLR signaling in a manner consistent with a role in insulin resistance, based on analogous studies in immune cells. Among them, IRF3 is activated by the MyD88-independent arm of TLR4 signaling via phosphorylation by the upstream 
A

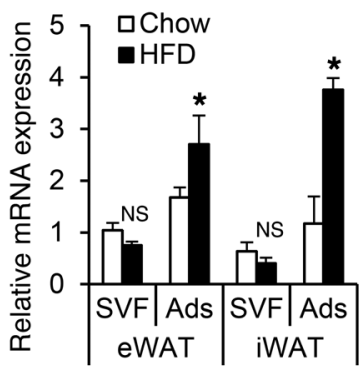

C

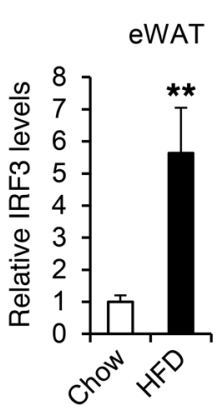

$\mathbf{F}$

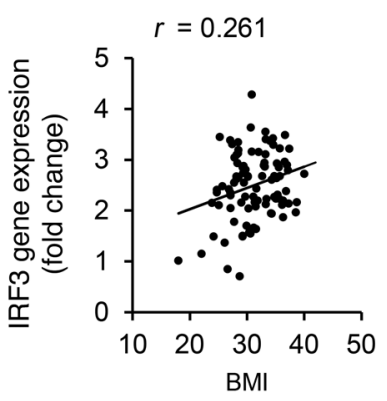

B

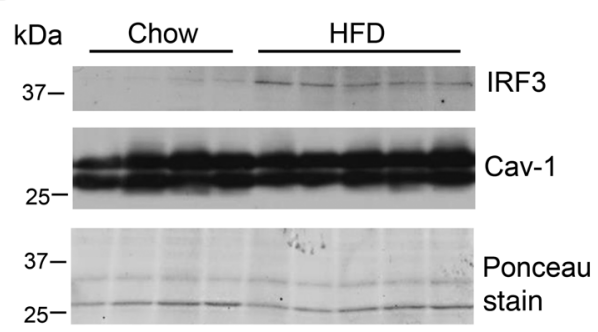

\section{D}

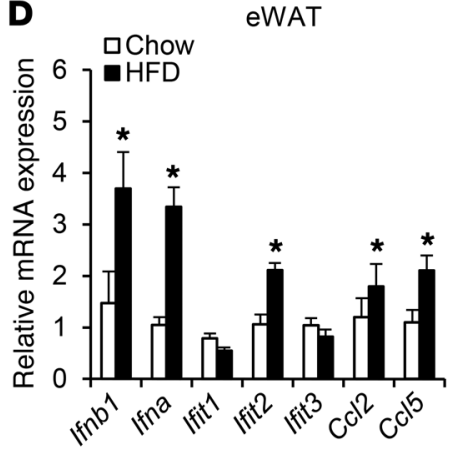

G

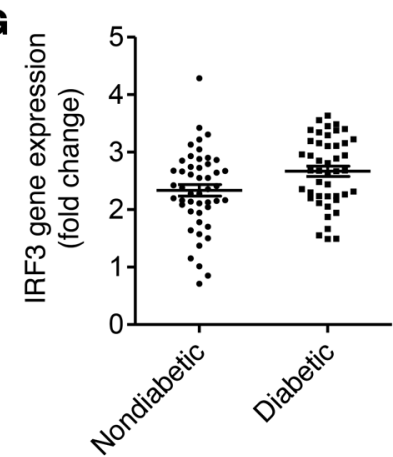

IWAT

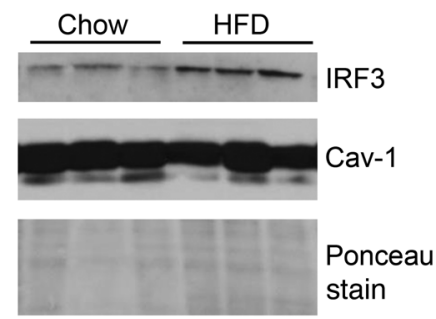

E

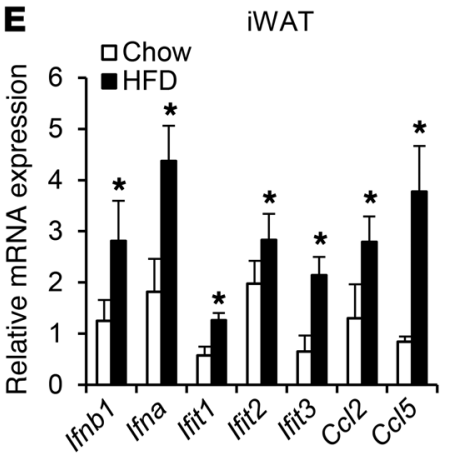

Figure 1. Elevated IRF3 levels in adipose tissue of obese mice and humans. (A) Irf3 mRNA levels were measured in male mice fed chow versus high-fat diet (HFD) by qPCR in isolated stromal-vascular and adipocyte fractions from eWAT and iWAT. ${ }^{*} P<0.05$. (B) Western blot showing IRF3 protein levels in isolated adipocytes from eWAT and iWAT of high-fat- and chow-fed mice. (C) Quantification of the blots in $\mathbf{B}$. ${ }^{*} P<0.01$. (D and E) Expression of known IRF3 target genes in isolated adipocytes from eWAT (D) and iWAT (E) of chow- and HFD-fed mice by qPCR. ${ }^{*} P<0.05$. Data are presented as mean \pm SEM, $n=8$ per group. (F) IRF3 mRNA expression correlates with BMI in human subjects. $n=93, P=0.011$. (C) IRF3 mRNA expression in nondiabetic $(n=48)$ and diabetic $(n=45)$ humans. $P=0.015$.

kinases TBK1 and IKK- $\varepsilon$, resulting in IRF3 dimerization, nuclear translocation, and DNA binding $(16,20)$. Interestingly, levels of TBK1 and IKK- $\varepsilon$ are both elevated in the adipose tissue of obese mice, and inhibition of these kinases by genetic or pharmacological means improves systemic insulin sensitivity $(21,22)$.

Here, we show that IRF3 expression is elevated in the adipose tissue of obese rodents and humans. We also demonstrate that IRF3 is a requisite downstream effector of insulin resistance in response to activated TLR signaling, and that this effect is cell autonomous. Consistent with this, $\operatorname{Irf} 3^{-/-}$mice are protected from insulin resistance in the setting of high-fat feeding. Knockout mice show reduced inflammation within the adipose tissue and systemically, and enhanced glucose uptake specifically in adipose tissue. Furthermore, $\operatorname{Irf3} 3^{--}$mice have enhanced thermogenesis due solely to browning of subcutaneous fat, with no alterations seen in interscapular brown adipose tissue. These studies identify IRF3 as a major transcriptional effector of insulin resistance and the inflammatory response associated with overnutrition.

\section{Results}

IRF3 expression is elevated in adipocytes from obese mice and humans. To determine whether IRF3 plays a role in obesity-associated dysfunction of adipose tissue, we began by asking whether IRF3 expression is elevated in the fat cells of obese mice. In order to avoid confounding by infiltrating macrophages, we separated mature adipocytes from the stromal-vascular fraction (SVF) of inguinal and epididymal adipose tissue of high-fat-fed mice. Irf3 mRNA levels were increased in the adipocyte fraction from both depots; interestingly, we found a nonsignificant reduction in Irf3 in the SVF of obese mice (Figure 1A), despite clear evidence of increased macrophage content (Supplemental Figure 1, A and B; supplemental material available online with this article; doi:10.1172/JCI86080DS1). These findings were confirmed in isolated $\mathrm{F} 4 / 8 \mathrm{O}^{+} \mathrm{CD}_{11 \mathrm{~b}^{+}}$macrophages from inguinal and epididymal fat of chow- and high-fat-fed mice (Supplemental Figure 1, C and D). IRF3 protein levels were also elevated 5.6-fold in the adipocyte fraction of the epididymal fat pad and 
A $\simeq$

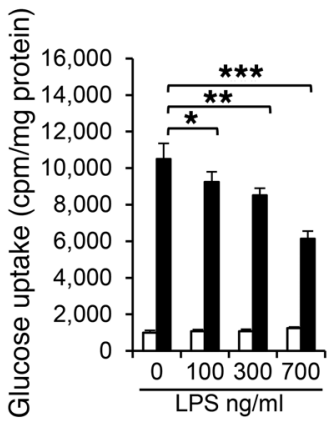

D

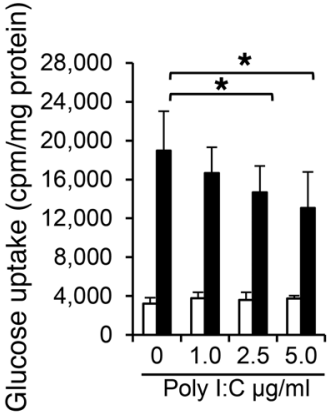

B

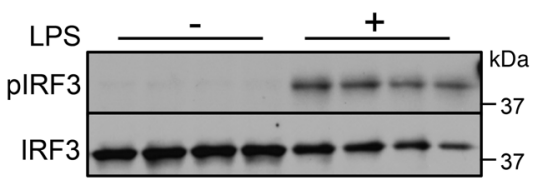

C

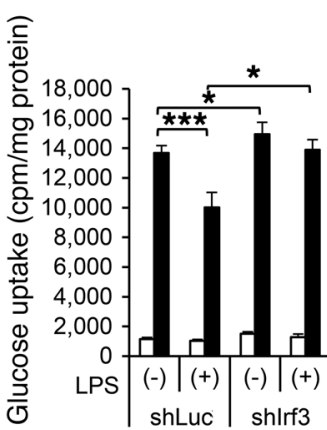

$\mathbf{E}$

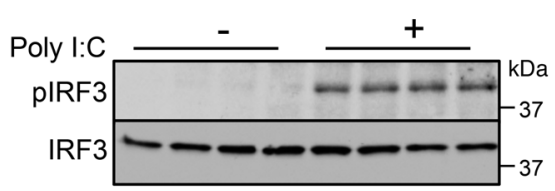

$\mathbf{F}$

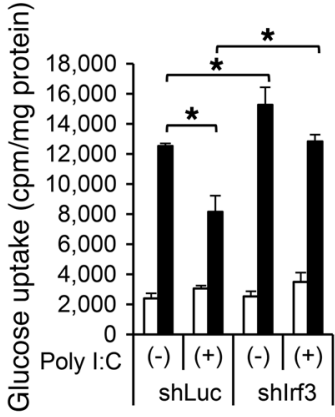

口Basal

Insulin

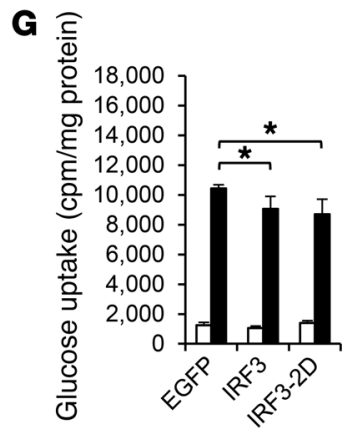

Figure 2. IRF3 regulates insulin sensitivity in adipocytes in a cell-autonomous fashion. (A) Basal and insulin-stimulated glucose uptake in 3T3-L1 adipocytes after treatment with varying doses of LPS for 6 days. (B) Western blot showing phosphorylation of murine IRF3 (Ser388) in 3T3-L1 adipocytes after 6 days of LPS $(700 \mathrm{ng} / \mathrm{ml})$ treatment. (C) Basal and insulin-stimulated glucose uptake in 3T3-L1 adipocytes transduced with lentivirus expressing shRNA against Irf3 or shLuc control hairpin in the absence or presence of LPS treatment $(700 \mathrm{ng} / \mathrm{ml})$. (D) Basal and insulin-stimulated glucose uptake in 3T3-L1 adipocytes after treatment with varying doses of poly I:C. (E) Western blot showing phosphorylation of IRF3 in 3T3-L1 adipocytes after 24 hours of poly I:C (5 $\mathrm{g} / \mathrm{ml})$ treatment. (F) Basal and insulin-stimulated glucose uptake in 3T3-L1 adipocytes transduced with lentivirus expressing shRNA against Irf3 or shLuc control hairpin in the absence or presence of poly I:C treatment $(5 \mu \mathrm{g} / \mathrm{ml})$. (G) Basal and insulin-stimulated glucose uptake in 3T3-L1 adipocytes expressing WT IRF3 or IRF3-2D mutant. Data in all panels expressed as mean \pm SEM. ${ }^{*} P<0.05,{ }^{* *} P<0.01,{ }^{* * *} P<0.001$.

3.3-fold in the inguinal depot (Figure 1, B and C). Consistent with these findings, the expression of several known IRF3 target genes was upregulated in isolated adipocytes from the epididymal and inguinal white adipose tissue (eWAT and iWAT) of mice with diet-induced obesity (Figure 1, D and E). We next sought to determine whether IRF3 expression is elevated in the adipose tissue of obese humans. In subcutaneous fat, IRF3 mRNA levels were significantly correlated with increased BMI (Figure $1 \mathrm{~F})$. Furthermore, $I R F 3 \mathrm{mRNA}$ levels were increased in diabetic patients (Figure $1 G$ and Supplemental Table 1).

IRF3 mediates insulin resistance in response to LPS and polyinosinic-polycytidylic acid in cultured adipocytes. Despite a large literature showing associations between endotoxin, TLR4 signaling, and insulin resistance, there is a paucity of data demonstrating direct effects of LPS and TLR4 on insulin action in adipose tissue. To address this, we determined the effect of TLR4 activation on insulin-stimulated glucose uptake in mature 3T3L1 adipocytes. Chronic treatment with LPS led to a dose-dependent decrease in insulin-stimulated glucose uptake in these cells, indicating reduced insulin sensitivity (Figure 2A). LPS stimulation increased phosphorylation of murine IRF 3 at serine 388 (Figure $2 \mathrm{~B}$ ), known to promote nuclear translocation, dimerization, and binding to DNA (23). To determine whether IRF3 is required for LPS-stimulated insulin resistance, we knocked down IRF3 in mature adipocytes using lentiviral delivery of a specific shRNA
(Supplemental Figure 2A). This had the effect of restoring full insulin sensitivity to the LPS-treated cells (Figure 2C). Our regimen of chronic LPS administration is fully able to induce phosphorylation and nuclear translocation of NF- $\mathrm{\kappa B}$ (Supplemental Figure 2B), but does not increase the amount of NF-kB bound to DNA (Supplemental Figure 2C). Another TLR4 ligand, palmitate, was also able to cause insulin resistance in cultured adipocytes in a dose-dependent manner (Supplemental Figure 2D). As expected, knockdown of IRF3 also restored insulin sensitivity lost after palmitate exposure (Supplemental Figure 2E).

TLR3 signaling is another upstream activator of IRF3 (24), suggesting that TLR3 agonists (e.g., polyinosinic-polycytidylic acid [poly I:C]) might also promote insulin resistance in adipocytes. This was in fact the case (Figure 2D). Poly I:C treatment also robustly increased phosphorylation of IRF3 (Figure 2E). Although TLR3 activation was able to translocate NF- $\mathrm{kB}$ (Supplemental Figure $2 \mathrm{~F}$ ), RNAi-mediated reduction of IRF3 fully abrogated poly I:C-mediated insulin resistance (Figure 2F).

Finally, we speculated that IRF3 might be sufficient to cause insulin resistance, even in the absence of upstream TLR signaling. Mutation of Ser396 and Ser398 to Asp in human IRF3 results in a constitutively active allele without the need for external stimuli (25). We therefore generated the analogous double-mutant murine IRF3 allele (S388D/S390D; hereafter designated as IRF3-2D). Overexpression of the IRF3-2D mutant in 3T3-L1 adipocytes was 
A

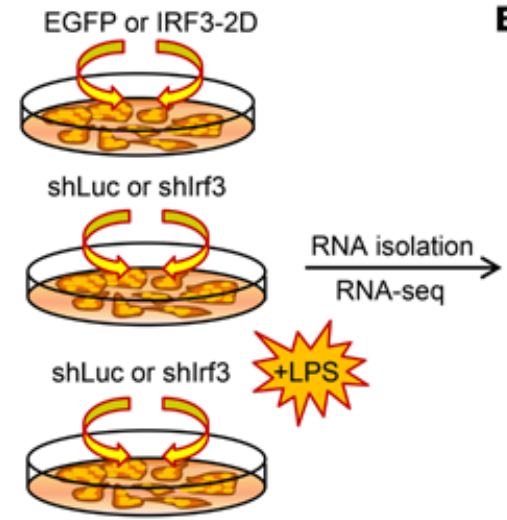

B

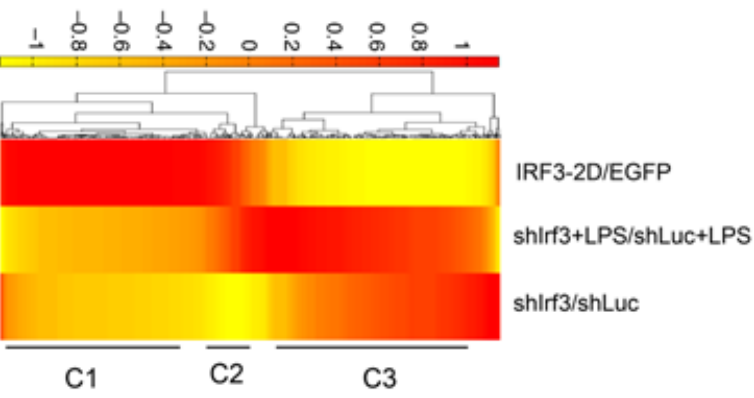

C

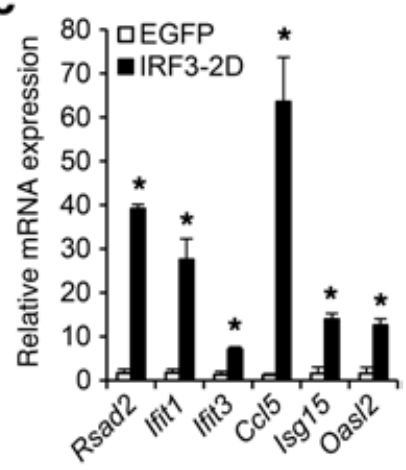

D

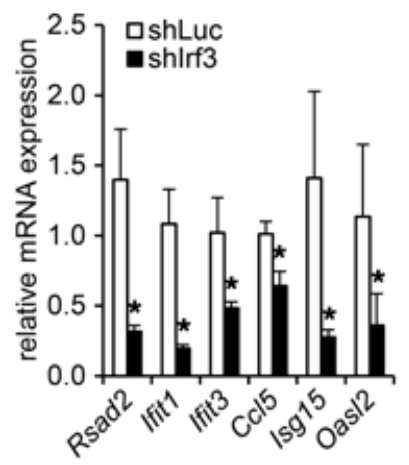

E

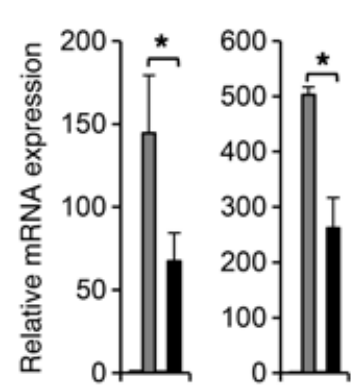

Serpina3g

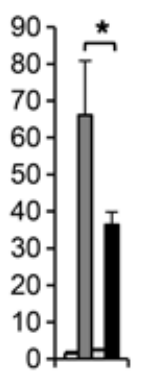

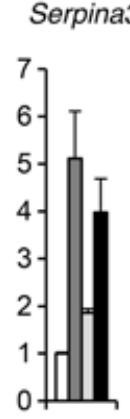

Tmem176a Tmem176b Ccl6
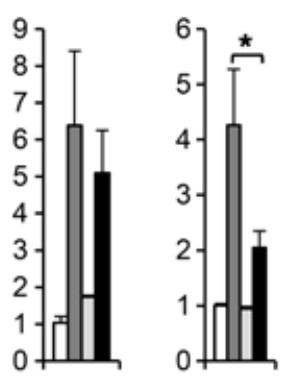

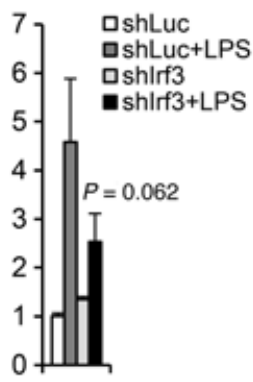

Figure 3. IRF3 regulates expression of immune genes in adipocytes. (A) Diagrammatic representation of the groups used for RNA-seq. (B) Heat map showing gene expression standardized fold change between 3 groups (IRF3-2D vs. EGFP, shlrf3 vs. shLuc, and shlrf3 vs. shLuc in the presence of LPS). Three different clusters identified are listed. Scale is $\log _{2}$ fold change. (C) mRNA expression of genes upregulated by IRF3-2D in 3T3-L1 adipocytes assessed by qPCR. (D) mRNA expression of genes from $\mathbf{D}$ was determined by qPCR after Irf3 knockdown in 3T3-L1 adipocytes. (E) mRNA expression of selected LPS target genes in the absence and presence of $/ r f 3$ knockdown as determined by qPCR. Data in all panels expressed as mean \pm SEM. ${ }^{*} P<0.05$.

confirmed using quantitative PCR (qPCR) and Western blotting (Supplemental Figure 3, A and B). In mature 3T3-L1 adipocytes IRF3-2D expression led to a robust increase in the expression of IRF3 target inflammatory genes, including Ifit1, $\mathrm{Ccl}$, and $\mathrm{Ccl} 5$ (Supplemental Figure 3C), showing that murine IRF3-2D mutant acts as a potent constitutively active IRF3 in adipocytes. Consistent with a role for IRF3 as a driver of insulin resistance, overexpression of IRF3-2D in mature adipocytes led to a significant decrease in insulin-stimulated glucose uptake in the absence of upstream activators (Figure 2G). This was associated with diminished insulin-mediated phosphorylation of p70S6K at T389, without a concomitant effect on Akt phosphorylation (Supplemental Figure 3D).

IRF3 is a transcriptional regulator of immune response genes in adipocytes. To further investigate the role of IRF3 in adipocytes, we performed transcriptional profiling in mature 3T3-L1 cells following lentiviral transduction of IRF3-2D (vs. an EGFP control) using RNA sequencing (RNA-seq). We then compared the resulting changes in gene expression to those seen following introduction of an shRNA against murine IRF3 (vs. an irrelevant hairpin control), in both the absence and the presence of LPS (Figure 3, A and B, and Supplemental Figure 4A). In general, the effect of IRF3-2D overexpression was stronger (557 genes upregulated, 505 genes downregulated at $\geq 1$. 4 -fold change, $25 \%$ FDR) than the effect of
IRF3 knockdown (271 genes upregulated, 376 genes downregulated) in the absence of LPS; this was expected given the fact that WT IRF3 needs to be activated by upstream TLR agonism (Supplemental Figure 4, B-F). Overall, 68 genes were coordinately regulated in the overexpression and knockdown models (45 genes up with IRF3-2D and down with shIRF3, 23 genes down with IRF3-2D and up with shIRF3) (Supplemental Table 2). These genes include IRF3 itself, of course, but also a large number of class I MHC genes (H2-Q1, H2-Q2, H2-Q6, H2-Q8, H2-K1, H2-K2, H2-Bl, H2-D1, H2Gs10, and H2-T23), all induced by IRF3-2D and repressed by shIRF3. This striking finding is consistent with the proposed role for adipocytes as antigen-presenting cells playing an early role in the inflammatory reaction to overnutrition $(6,26)$.

Gene set enrichment analysis identified genes from immunerelated pathways as among the strongest coordinately regulated groups (Supplemental Tables 3 and 4). Many of these genes are known direct or indirect targets of IRF3 in immune cells, suggesting that these same pathways exist in adipocytes. We confirmed the effect of IRF3 on inflammatory gene expression (e.g., Rsad2, Ifit1, Ifit3, Ccl5, Isg15, and Oasl2) by qPCR (Figure 3, C and D).

As expected, chronic LPS treatment exerts a very strong influence on gene expression, and most of these changes occurred regardless of whether shIRF3 or a control hairpin was present. 


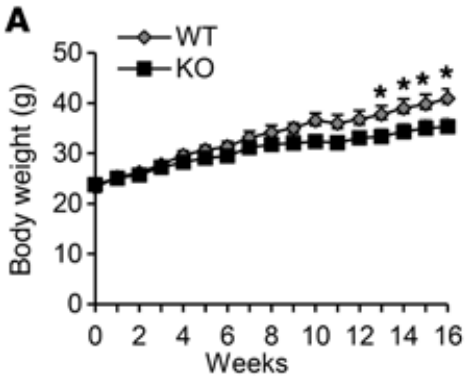

B
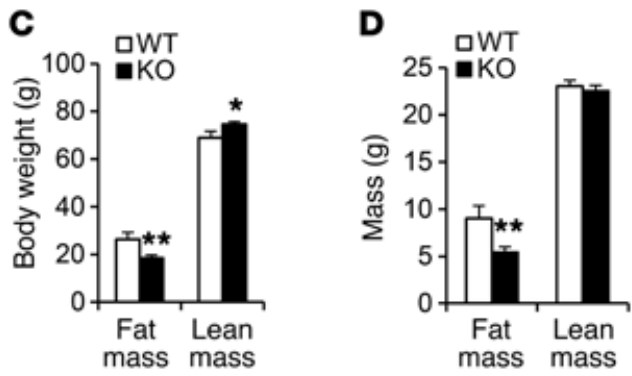
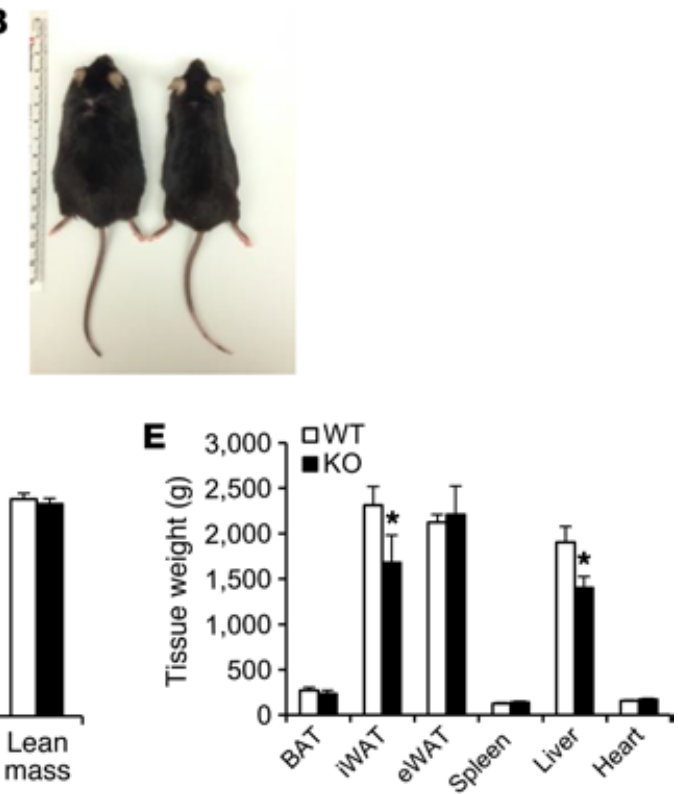

Figure 4. Irf3 ${ }^{-/-}$mice are protected from diet-induced obesity. Male WT and $/ \mathrm{rf3}^{-/ /}$ mice were fed HFD ( $58 \%$ fat wt/wt) for 16 weeks, $n=7-8$ per genotype. (A) Body weight of WT and $/ \mathrm{rf3}^{-/-}$mice during HFD feeding. (B) Gross morphology of WT and $/ \mathrm{rf3}^{-/-}$mice after 16 weeks on HFD. (C and D) Body composition of WT and Irf3 $3^{-/-}$mice at 12 weeks of HFD feeding. (E) Tissue weights of WT and $/ \mathrm{rf3}^{-/-}$mice after 16 weeks on HFD. Data are presented as mean \pm SEM, and experiments were repeated using 3 independent HFD cohorts. ${ }^{*} P<0.05,{ }^{* *} P<0.01$.
Nevertheless, we were able to identify several genes that were strongly induced by LPS in an IRF3-dependent manner. These include inflammatory genes (e.g., Ccl6, Nos2), in addition to genes that we have previously shown to cause insulin resistance in adipocytes (e.g., Tmem176b, Lcn2) (Figure 3E) (27).

Irf $3^{-/}$mice are modestly protected from diet-induced obesity and exhibit reduced adipose inflammation. Despite the well-established role of IRF3 in immunity, Irf3 $3^{-/-}$mice show a relatively mild immune phenotype (28). Consistent with this, we found modest reductions in inflammatory gene expression in the adipose tissue of chow-fed Irf $3^{-/-}$mice (Supplemental Figure 5A), which did not translate into significantly altered chemokine levels in the plasma of these animals (Supplemental Figure 5B). Irf3 $3^{-/-}$mice showed no significant difference in body weight or adiposity on chow diet (Supplemental Figure 5, C and D). This is consistent with the fact that IRF3 is inactive until stimulated by TLR signaling, which we would expect to be minimal in the lean state. We therefore exposed the mice to a high-fat diet to induce intra-adipose inflammation. Notably, Irf3 ${ }^{-/-}$mice displayed reduced fat mass and increased lean mass that became apparent after prolonged high-fat feeding (Figure 4, A-D). Specifically, the weight of iWAT and liver was significantly reduced in $\mathrm{Irf3}^{-/-}$mice on high-fat diet (Figure 4E).

As predicted from the in vitro data, the adipose tissues of Irf3 $3^{-/-}$mice have dramatically reduced inflammatory gene expres- sion after high-fat feeding than do WT littermates, associated with reduced circulating cytokine levels (Figure 5, A-C). Furthermore, both eWAT and iWAT of $\mathrm{Irf3}^{-1-}$ mice showed markedly reduced infiltration of $\mathrm{F} 4 / 80^{+}$cells on high-fat diet (Figure 6A). The total number of $\mathrm{F} 4 / 80^{+} \mathrm{CD} 11 \mathrm{~b}^{+}$macrophages was reduced in the SVF isolated from the eWAT of $\operatorname{Irf3} 3^{--}$mice, with a trend toward reduction in iWAT (Figure 6B). This was associated with a pronounced alteration in macrophage polarization state, with reduced numbers of M1 macrophages (CD11 $\mathrm{c}^{+}$) and increased M2 macrophages $\left(\mathrm{CD} 01^{+}\right)$in $\mathrm{Irf}^{-/-}$mice (Figure 6, C and D). These data suggest that IRF3 deficiency attenuates chronic diet-induced inflammation, both locally within adipose tissue and systemically.

IRF3 deficiency improves glucose homeostasis and insulin action. Our in vitro data indicate that IRF3 mediates the effect of inflammatory stimuli on insulin action in a cell-autonomous fashion, but we wished to determine whether this could be demonstrated in living animals. On chow diet, $\operatorname{Irf} 3^{-/-}$mice show no difference in plasma glucose, insulin, nonesterified free fatty acid (NEFA), or triglycerides (Supplemental Table 5). Glucose tolerance and insulin tolerance were also indistinguishable between WT and knockout mice on chow diet (Supplemental Figure 6, A and B). However, $\operatorname{Irf3} 3^{-/}$mice showed significantly reduced plasma insulin levels after high-fat feeding (Figure 7A) with unaltered plasma glucose levels (Supplemental Figure 6C). $\operatorname{Irf3}^{-/-}$mice on high-fat diet
Figure 5. Irf3 $^{-/-}$mice exhibit reduced adipose and systemic inflammation. Male WT and $\mathrm{Irf3}^{-1-}$ mice were fed HFD for 16 weeks, $n=7-8$ per genotype. (A and $\mathbf{B}$ ) Gene expression of IRF3 target genes in eWAT (A) and IWAT (B) of $/ \mathrm{rf3}^{-/-}$mice on HFD. (C) Plasma cytokine levels in Irf3 $3^{-1-}$ mice on HFD. Data are presented as mean \pm SEM. For all data, ${ }^{*} P<0.05$.

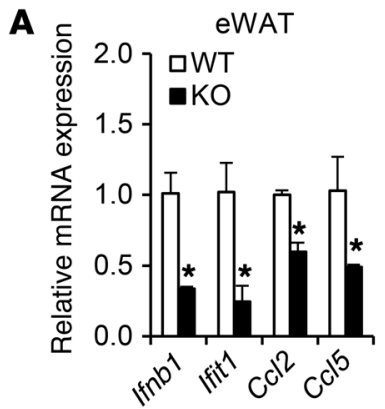

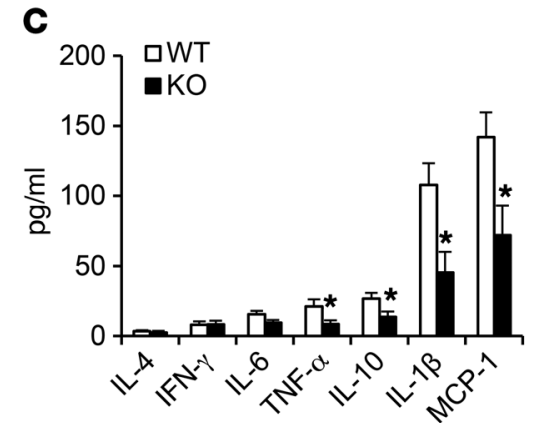


A

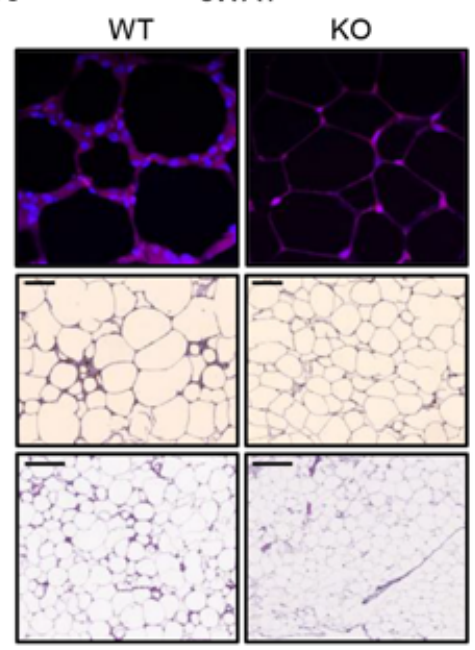

IWAT

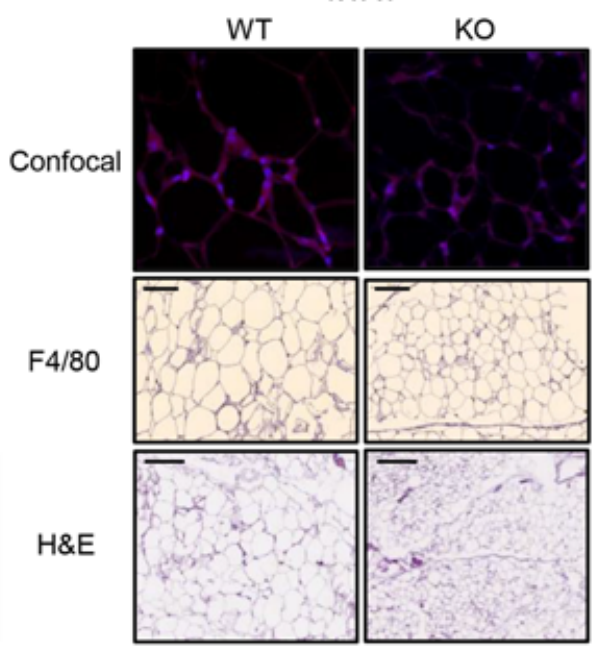

B

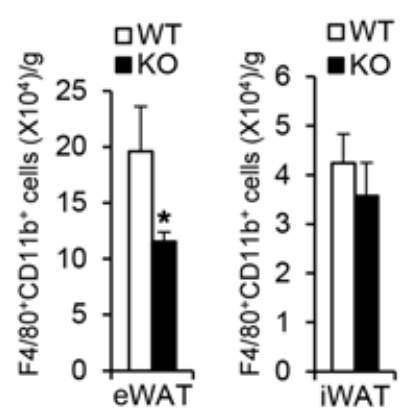

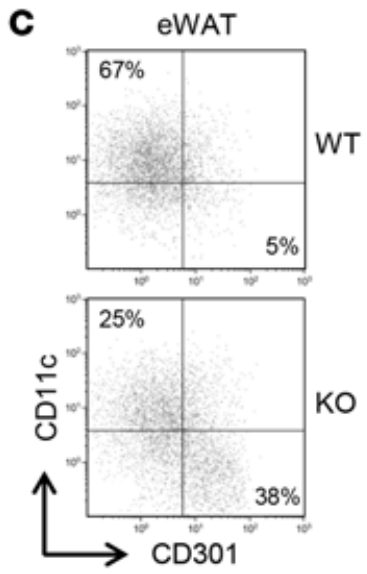
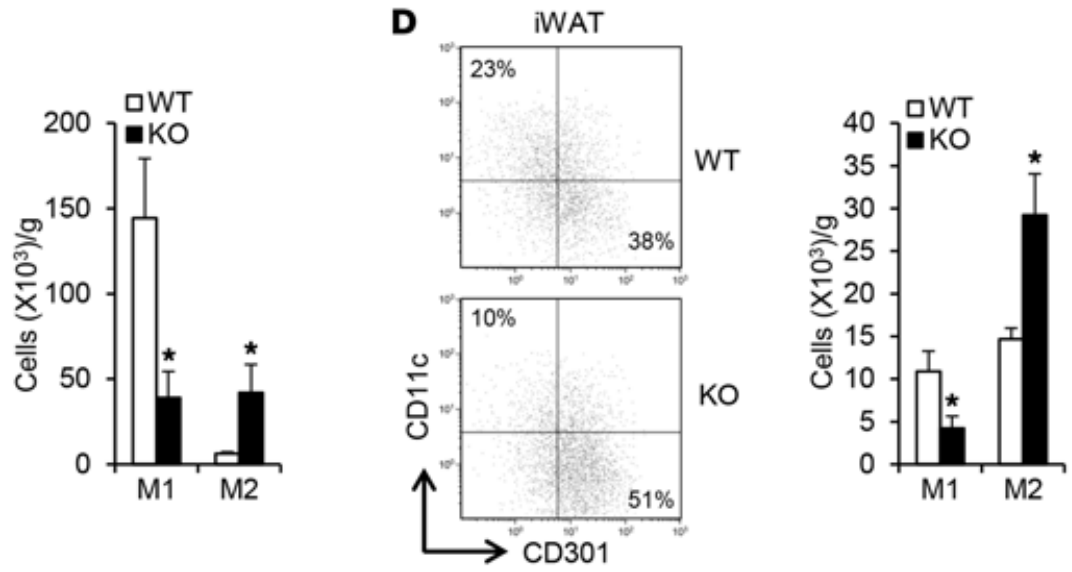

Figure 6. Irf3 $3^{-/-}$mice exhibit reduced infiltration and altered polarization of macrophages in adipose. Male WT and Irf $3^{-/-}$mice were fed HFD for 16 weeks, $n=7-8$ per genotype. (A) Histology of eWAT and iWAT from WT and $/ \mathrm{rf3}^{-1-}$ mice, including F4/80 immunostaining. Confocal images were taken at $\times 40$ magnification. For F4/80 immunostaining, scale bars $=100 \mu \mathrm{m}$, and for $\mathrm{H} \& \mathrm{E}$ staining, scale bars $=200 \mu \mathrm{m}$. (B) Adipose tissue macrophage

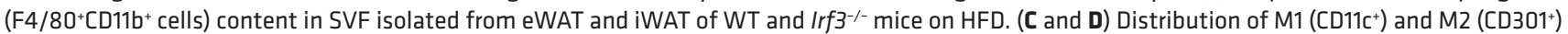
macrophages in SVF from eWAT (C) and iWAT (D) of WT and Irf3 ${ }^{-/-}$mice on HFD. Data are shown as absolute number normalized to fat pad weight. $n=5$ per genotype. Data are presented as mean $\pm \mathrm{SEM}$. For all data, ${ }^{*} P<0.05$.

exhibited no change in plasma triglyceride or NEFA levels (Supplemental Table 5). Interestingly, Irf $3^{-1-}$ mice showed improved glucose and insulin tolerance after 8 and 10 weeks of high-fat feeding, time points at which body weight was not significantly different between the 2 genotypes (Figure 7, B and C).

We next asked whether improved glucose homeostasis in Irf3-mice is reflected in improved insulin signaling. Indeed, phosphorylation of Akt (S473) and p70S6K (T389) was significantly increased in iWAT of Irf3- mice on high-fat diet (Figure 7, D and E). In addition, eWAT and liver of $\operatorname{Irf3}^{---}$mice also showed modest increases in insulin-stimulated phosphorylation of Akt (S473) (Supplemental Figure 6, D-G).

In order to confirm that the differences in glucose and insulin tolerance seen at 8-10 weeks of high-fat feeding did not reflect very small changes in adiposity between WT and Irf $3^{-/}$mice, we repeated these studies on a new cohort of mice after only 6 weeks of high-fat feeding, at which point adiposity was clearly equivalent between WT and $\mathrm{Irf3}^{-/-}$mice. Again, glucose and insulin tolerance testing revealed improved insulin sensitivity in Irf3-- mice (Figure 7, F and G).

Increased glucose uptake in inguinal fat of $\mathrm{Irf3}^{-/-}$mice. We also subjected Irf3 $3^{-/}$mice to hyperinsulinemic-euglycemic clamping to assess tissue-specific insulin sensitivity. Again, to dissociate effects on insulin action from changes in adiposity, we studied animals before body weights diverged (Figure 8A). There was a pronounced increase in glucose infusion rate at steady state in Irf3 $3^{--}$mice, indicating improved insulin sensitivity (Figure 8B). Plasma glucose and insulin levels were not altered between these groups (Supplemental Figure 7, A and B). Unaltered NEFA levels indicated that insulin-mediated suppression of lipolysis was not affected by the absence of IRF3 (Supplemental Figure 7C). Although Irf3 $3^{-/}$mice show slightly decreased basal glucose production, under clamp conditions hepatic glucose production was not different (Figure 8C). Similarly, glucose turnover was reduced in $\operatorname{Irf} 3^{-/}$mice under basal, but not under clamp, conditions (Figure 8D). In vivo glucose 


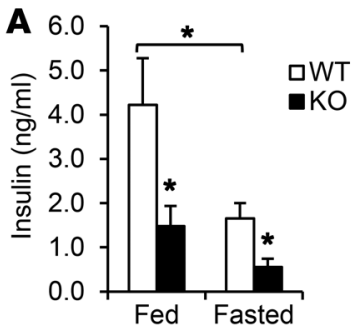

D

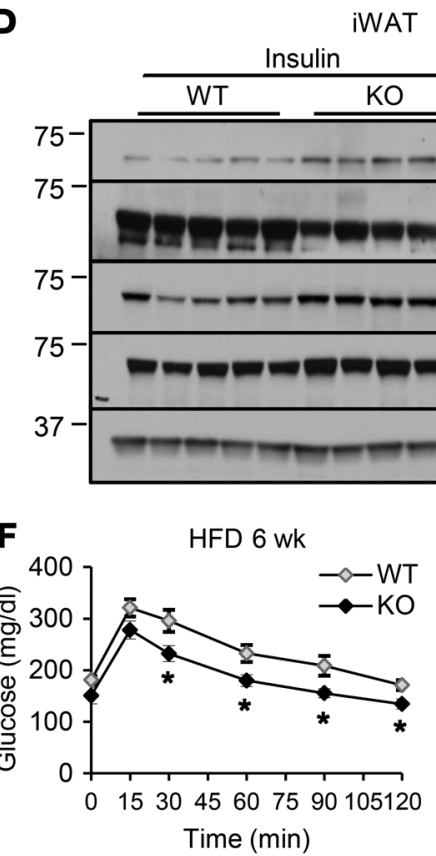

B

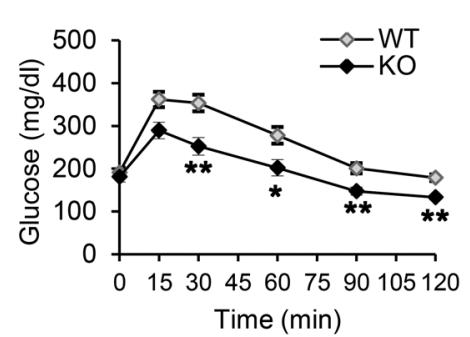

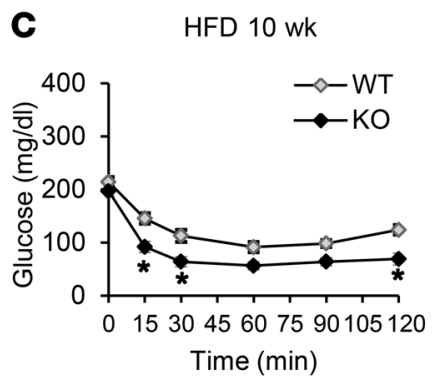

$\mathbf{E}$

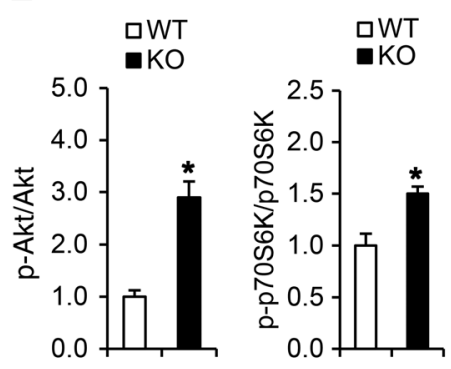

Figure 7. Irf $3^{-/-}$mice show improved insulin sensitivity on HFD. (A) Fed and fasting plasma insulin levels in WT and Irf3 $3^{-/-}$mice on 16 weeks of HFD ( $n=7-8$ per genotype). (B) Glucose tolerance test after 8 weeks on HFD. (C) Insulin tolerance test after 10 weeks on HFD. (D) Insulin-stimulated Akt and p70S6K phosphorylation in iWAT of WT and Irf3-/- mice after 16 weeks on HFD. (E) Quantification of Western blot in D. (F) Glucose tolerance testing of WT and Irf3 $3^{-/-}$mice after 6 weeks of HFD, prior to weight divergence. (C) Insulin tolerance testing of WT and Irf $3^{-1-}$ mice after 8 weeks of HFD, prior to weight divergence. Data are presented as mean $\pm \mathrm{SEM}$. ${ }^{*} P<0.05,{ }^{* *} P<0.01,{ }^{* *} P<0.001$ for all panels.

uptake was significantly increased specifically in iWAT, but not in eWAT, brown adipose tissue (BAT), or muscle (Figure 8, E and F).

Increased GLUT4 levels in adipose tissue of $\operatorname{Irf} 3^{-1-}$ mice. We first hypothesized that IRF3 modulates glucose homeostasis via the transcriptional regulation of genes involved in insulin action. One such target suggested by our RNA-seq data was adiponectin (encoded by the Adipoq gene). Consistent with this, Adipoq expression was repressed in adipocytes exposed to IRF3-2D, and increased in cells with IRF3 knockdown (Supplemental Figure 8A). This pattern was recapitulated in vivo, with increased Adipoq expression in the WAT of $\operatorname{Irf3}^{-/-}$mice (Supplemental Figure 8B). Surprisingly, however, plasma adiponectin levels did not differ between WT and knockout mice (Supplemental Figure 8C), suggesting that this change in gene expression does not account for the insulin sensitization observed in $\mathrm{Irf3}^{-/-}$mice. Another IRF3 target gene that we have previously identified (19) is $S l c 2 a 4$, encoding GLUT4. Obese WT mice show reduced adipose GLUT4 protein levels in eWAT and iWAT (29) (Supplemental Figure 8D). Overexpression of IRF3 in 3T3-L1 adipocytes led to decreased Slc2a4 mRNA, and IRF3 knockdown resulted in the opposite effect (Sup- plemental Figure 8E). Concordant with this, Slc2a 4 mRNA and GLUT4 protein levels were increased in the eWAT and iWAT (but not muscle) of $\mathrm{Irf3}^{-/-}$mice on high-fat diet (Figure 9, A-C).

Irf $3^{-/}$mice exhibit increased beige fat recruitment in iWAT. TLR4 stimulation by LPS has been implicated as a cause of the reduced adaptive thermogenesis that accompanies high-fat feeding and obesity (30). We wondered whether the resistance to diet-induced obesity and increased subcutaneous adipose glucose uptake could be due to enhanced browning. This possibility gained support when we noted that $\mathrm{Irf3}^{-1-}$ mice on high-fat diet ate approximately $0.4 \mathrm{~g} / \mathrm{d}$ more than WT mice (Supplemental Figure 9A), indicating that reduced food intake is not the cause of the reduced adiposity. Similarly, $\mathrm{Irf3}^{-/-}$and WT mice evinced no difference in physical activity (Supplemental Figure 9B). However, Irf3 $3^{--}$mice did exhibit increased oxygen consumption, carbon dioxide production, and heat output and a reduced respiratory exchange ratio (Figure 10, A and B, and Supplemental Figure 9, C and D). Taken together, these results suggest that IRF3 deficiency promotes enhanced energy expenditure through a non-activity-based mechanism. Inguinal adipose tissue has the potential to develop increased numbers of $\mathrm{UCP}^{+}$ 
A

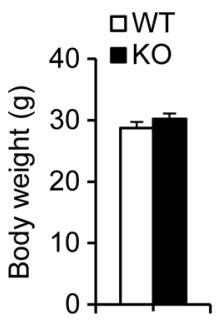

B

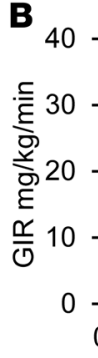

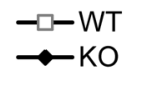

\section{T}

西
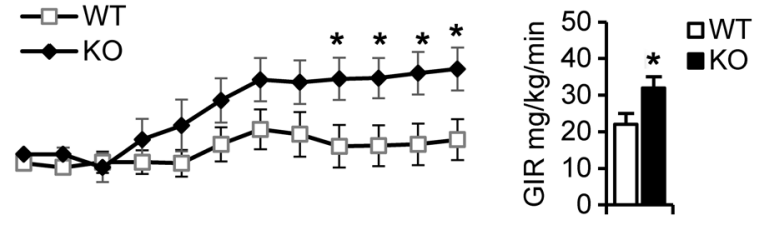
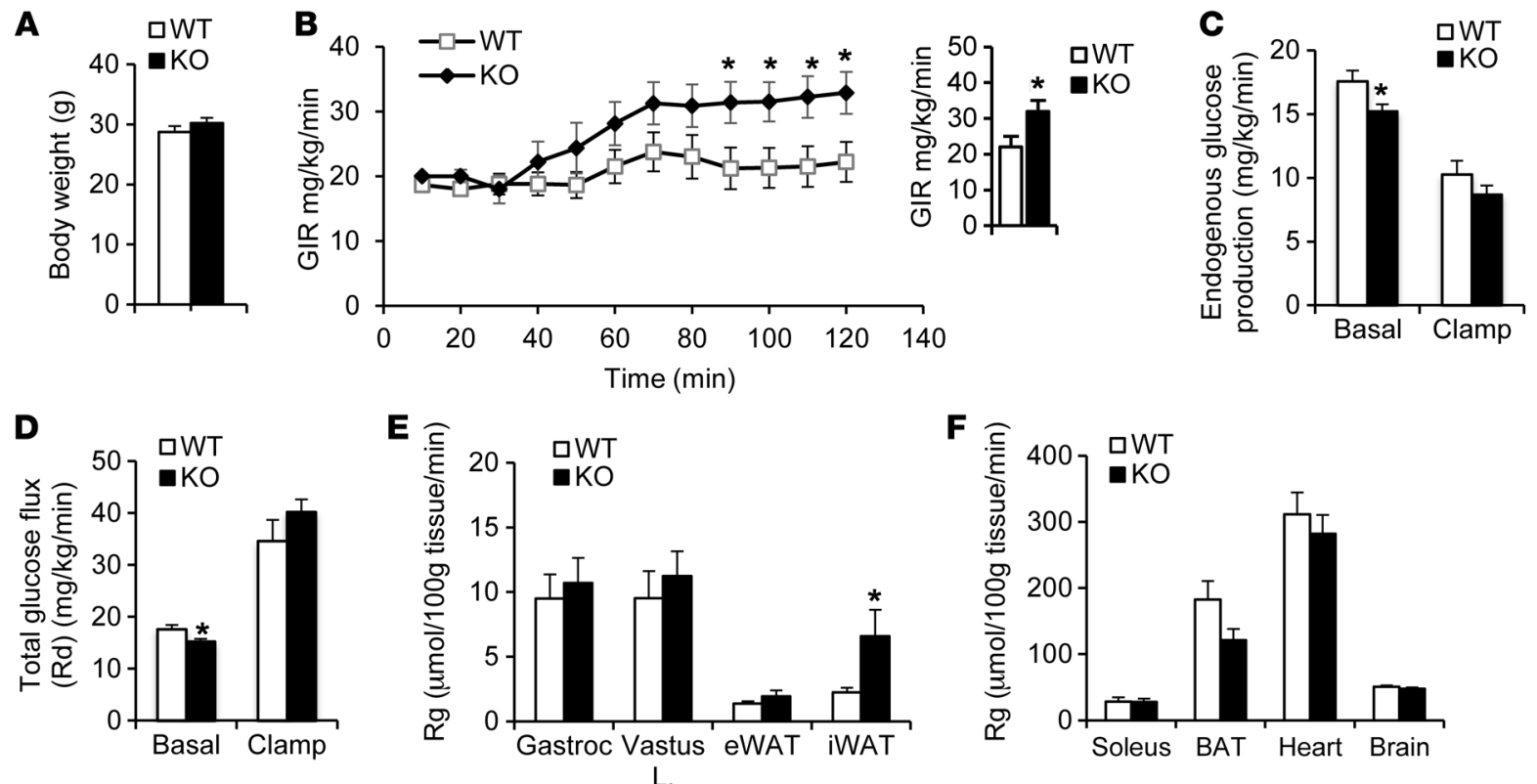

Figure 8. Irf3 $3^{-/-}$mice show increased glucose uptake in iWAT. WT and $/ \mathrm{rf}^{-/-}$mice ( $n=8-10$ per genotype) were treated with HFD for 8 weeks and then subjected to hyperinsulinemic-euglycemic clamping. (A) Body weight of mice at time of clamp. (B) Glucose infusion rate (GIR) over the course of the clamp; inset graph shows difference in the steady-state (80-120 minutes) GIR between WT and $/ \mathrm{rf3}^{-/-}$mice. (C) Endogenous glucose production before and during insulin infusion. (D) Total glucose flux (Rd) before and during insulin infusion. (E and F) Tissue-specific glucose metabolic index (Rg) in WT and Irf3 ${ }^{-/-}$mice under clamp conditions. Data are presented as mean \pm SEM. ${ }^{*} P<0.05$ for all data.

beige adipocytes, which dissipate energy via enhanced thermogenesis (31). This was further suggested by the selective reduction in inguinal fat pad weight noted earlier (Figure 4E). In fact, histological analysis revealed increased $\mathrm{UCP}^{+}$cells and increased expression of thermogenic marker genes in iWAT of $\mathrm{Irf3}^{-/-}$mice on high-fat diet (Figure 10, C and D). There was no significant effect on histology or gene expression of thermogenic genes in interscapular BAT (Supplemental Figure 9, E and F). Consistent with this, oxygen consumption was increased in the iWAT, but not the BAT, of $\operatorname{Irf} 3^{-/-}$mice (Figure 10E). Interestingly, $\mathrm{Irf3}^{-/-}$mice still displayed increased expression of thermogenic markers in iWAT even after 1 month at thermoneutrality, suggesting that IRF3 acts a temperature-independent "brake" on browning (Figure 11A). In order to determine whether the antithermogenic actions of IRF3 are cell autonomous, we differentiated SVF from iWAT of $\mathrm{Irf3}^{-/-}$mice ex vivo, and demonstrated enhanced ther- mogenic gene expression (Figure 11B). Furthermore, overexpression of IRF3 or IRF3-2D by adenoviral transduction in mature 3T3-F442A cells led to significant downregulation of thermogenic markers in a cell-autonomous manner (Figure 11C).

Notably, repeating the energy expenditure experiments in $I r f 3^{-/-}$ mice on high-fat diet prior to weight divergence (Supplemental Figure $10, \mathrm{~A}-\mathrm{C}$ ) yielded the same effect on $\mathrm{O}_{2}$ consumption and $\mathrm{CO}_{2}$ production (Figure 12, A and B), again without effect on food intake, activity, respiratory exchange ratio, or heat production (Supplemental Figure 10, D-G). Similarly, these mice showed increased UCP1 ${ }^{+}$ immunostaining and increased expression of thermogenic markers in the iWAT (Figure 12, C and D), but no change in histology or gene expression of thermogenic genes in interscapular BAT, although we noted a small but significant increase in genes associated with fatty acid oxidation (Supplemental Figure 10, $\mathrm{H}$ and I).
A

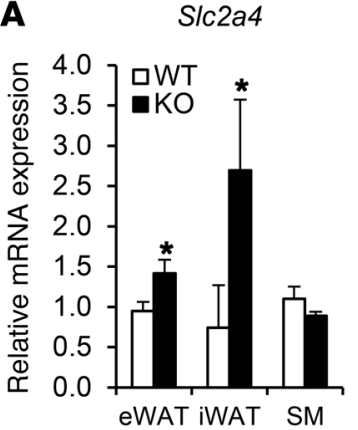

B

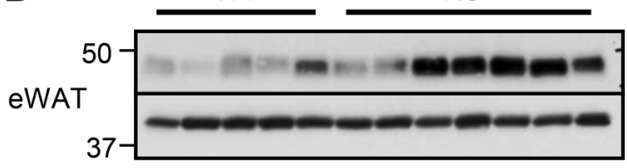

GLUT4 $\beta$-Actin

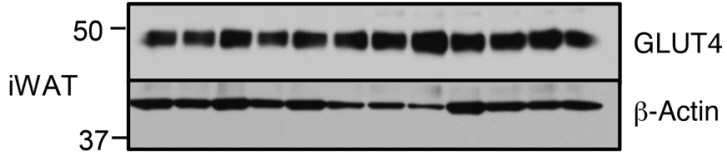

\section{GLUT4}

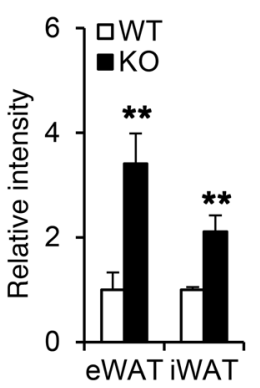

Figure 9. Irf3-1- mice show increased GLUT4 levels in adipose tissue. (A) Slc2a4 mRNA levels in eWAT, iWAT, and skeletal muscle (SM) of WT and Irf3 ${ }^{-/-}$ mice on 16 weeks of HFD, $n=7-8$ per genotype. (B) GLUT4 protein levels in eWAT and iWAT of WT and Irf3 ${ }^{-/-}$mice on 16 weeks of HFD. (C) Quantification of Western blot in $\mathbf{B}$. Data are presented as mean \pm SEM. ${ }^{*} P<0.05,{ }^{* *} P<0.01$ for all data. 
A

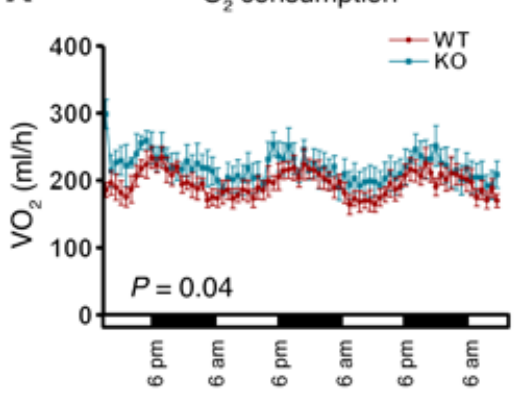

B

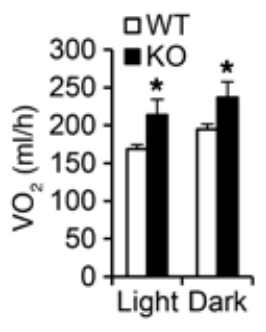

$\mathrm{CO}_{2}$ production

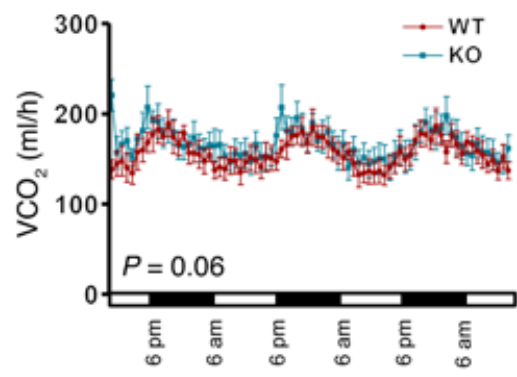

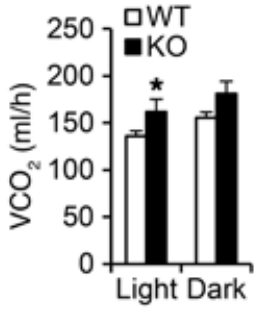

C

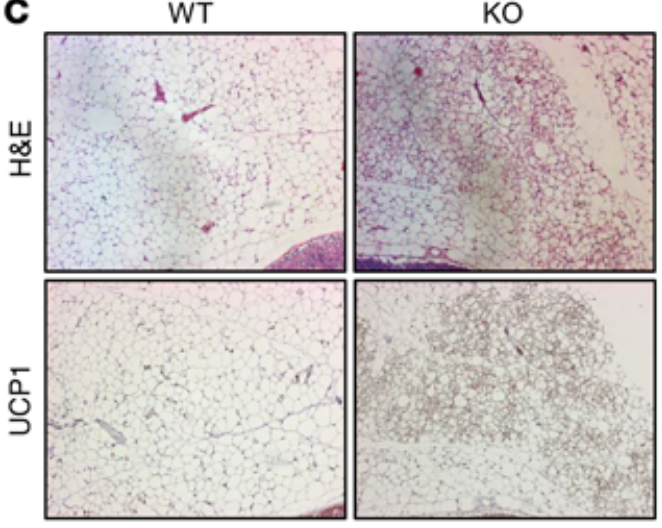

D

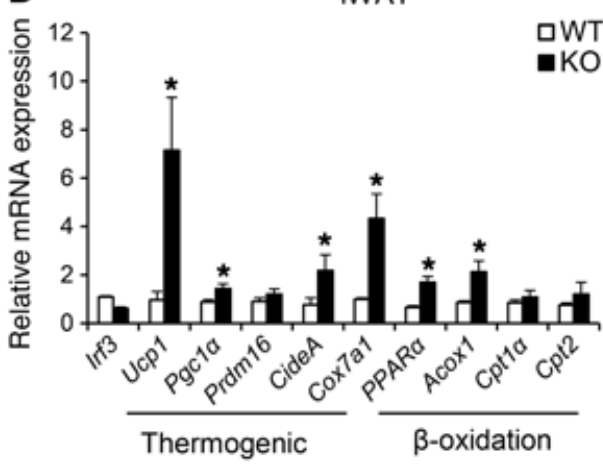

E

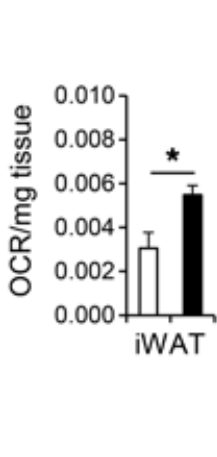

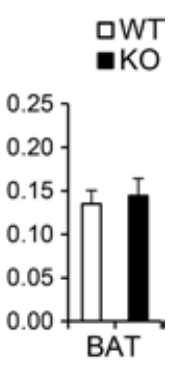

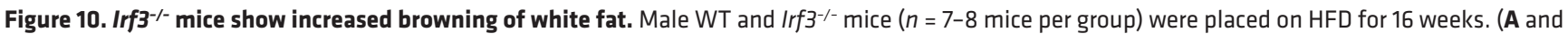
B) Oxygen consumption $\left(\mathrm{VO}_{2}\right)(\mathbf{A})$ and carbon dioxide production $\left(\mathrm{VCO}_{2}\right)(\mathbf{B})$ at day and night cycle were determined by CLAMS. (C) Histology showing H\&E and UCP1 immunostaining in iWAT of WT and $/ \mathrm{rf3}^{-1-}$ mice on HFD (original magnification, $\times 10$ ). (D) Expression of thermogenic and $\beta$-oxidation genes in iWAT of WT and Irf3 $3^{-1-}$ mice on HFD. (E) Oxygen consumption rate (OCR) in iWAT and BAT of WT and Irf3 $3^{-/-}$mice on chow diet. $n=4$ per genotype. Data are presented as mean \pm SEM. For all data, ${ }^{*} P<0.05$.

\section{Discussion}

Chronic low-grade inflammation, particularly within the adipose depot, is a sine qua non of obesity and a driving factor in local and systemic insulin resistance $(4,32)$. Numerous studies have detailed changes in the cellular composition of the immune compartment within adipose tissue in response to overnutrition, and many cytokines and other soluble mediators of inflammation have been implicated in the metabolic dysfunction that characterizes obesity (33). In this study, we have identified IRF3 as a key early transcription factor in the response of adipocytes to high-fat feeding, coordinating inflammation, macrophage infiltration, browning, and insulin sensitivity.

The earliest steps in the inflammatory cascade have been difficult to elucidate. One theory holds that adipocyte hypertrophy produces local hypoxia within the fat pad, which drives the inflammatory changes characteristic of obesity (34-36). Alternatively, caloric excess results in altered mitochondrial respiration, which in turn activates a host of stress-related pathways including oxidative and ER stress; these pathways can initiate inflammatory changes in adipocytes and reduce insulin action (37-40). Another, non-mutually exclusive, hypothesis is that innate immune pathways within adipocytes are directly stimulated by nutrients or other circulating factors, and this triggers the influx of inflammatory cells. In this last scenario, the TLRs are likely to play a pivotal role, as they are key sensors of foreign pathogens and cellular stress. TLR4 in particular is activated by free fatty acids (FFAs) and by LPS, both of which circulate in higher than normal quantities in the obese state (41-43). Thus, mice lacking TLR4 become obese on a high-fat diet, but are protected from insulin resistance, and TLR4 expression and activity are elevated in the adipose tissue of both obese rodents and humans $(5,9)$. It thus seems reasonable to propose that TLR signaling mediates at least some of the effect of overnutrition in adipocytes.

In immune cells, TLR4 signaling proceeds through 2 parallel pathways, 1 of which is dependent on the actions of the adaptor protein MyD88, culminating in the activation of IKK- $\beta$ and NF- $\kappa B$. There is also a MyD88-independent pathway, which involves TRIF/TRAM and other adaptors, and leads to activation of the kinases TBK1 and IKK-ع. These kinases act on substrates including the transcription factor IRF3, which then dimerizes, translocates to the nucleus, and binds to DNA. Importantly, IRF3 and the MyD88dependent arm of signaling NF- $\mathrm{BB}$ activate distinct but overlapping sets of target genes, and in some cases may cooperate physically and functionally to induce inflammatory gene expression $(44,45)$.

Despite the well-known involvement of IRF3 as a mediator of TLR-induced (in particular, TLR3 and TLR4) gene expression changes, the focus of many in the community has centered on NF- $\kappa \mathrm{B}$ as the primary transcriptional driver of inflammatory gene expression in the adipocyte (33). It is true that activation of $\mathrm{NF}-\kappa \mathrm{B}$ can be detected in adipocytes from obese, insulin-resistant 

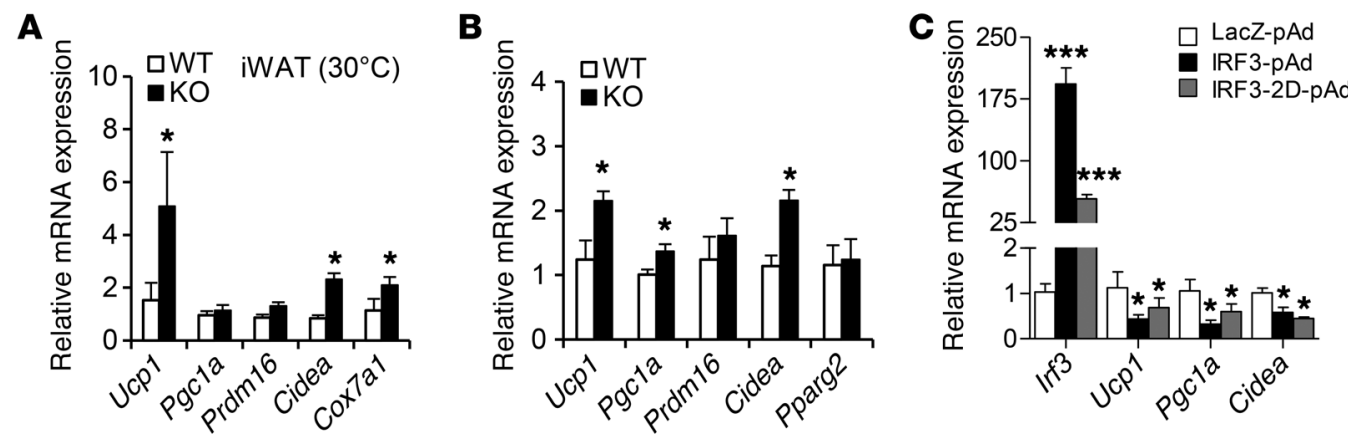

Figure 11. IRF3 represses thermogenic genes at thermoneutrality and in a cell-autonomous manner. (A) Expression of thermogenic genes in iWAT of Irf $3^{-1-}$ on chow diet at thermoneutrality $\left(30^{\circ} \mathrm{C}\right)$ for a month. $n=8-10$ per genotype. (B) Expression of thermogenic genes in ex vivo differentiated adipocytes from SVF of iWAT of WT and Irf3 $3^{-1-}$ mice. (C) Expression of thermogenic genes in 3T3-F442A adipocytes expressing WT IRF3 or IRF3-2D mutant after adenoviral transduction. Data are presented as mean \pm SEM. For all data, ${ }^{*} P<0.05,{ }^{* *} P<0.001$.

animals (21), but this does not prove that NF- $\mathrm{BB}$ is causal. Studies that manipulate IKK- $\beta$ activity in liver have indirectly suggested a role for NF- $\mathrm{KB}$, such that IKK- $\beta$ overexpression promotes insulin resistance, while animals expressing an IкB "super-repressor" are more insulin sensitive (12). Interestingly, these same manipulations in skeletal muscle promoted (or inhibited, in the case of the super-repressor) atrophy, without necessarily affecting insulin action. Furthermore, activation of IKK- $\beta$ in the mediobasal hypothalamus or in AGRP-containing neurons promotes obesity and metabolic dysfunction (46). Thus, the role of IKK- $\beta$ in metabolic dysfunction varies in a tissue-specific manner; unfortunately, analogous studies have not been described for adipose tissue. Notably, Scherer's group has shown that, in distinction to the situation in preadipocytes, NF- $\mathrm{KB}$ becomes unresponsive to LPS in mature adipocytes (11). IKK- $\beta$ manipulation presents an additional problem, in that we now understand that NF-кB is not the only target of this kinase. In fact, such targets include proteins like IRS-1, which could easily explain the effects of IKK- $\beta$ on insulin action (14). Direct targeting of NF- $\mathrm{\kappa B}$ in adipose tissue has been achieved, and the results do not support a major role for this factor in mediating insulin resistance due to inflammation. Ablation of p65, the major active subunit of NF-кB, using an aP2-driven Cre transgene causes reduced adipose inflammation in the lean state, but greater inflammatory markers upon high-fat feeding. No change in insulin sensitivity was noted between these knockout mice and controls on high-fat diet (13). Similarly, p65 overexpression in adipocytes induces browning of white fat, which promotes insulin sensitivity, not insulin resistance (15).

IRFs play a variety of roles as downstream effectors of inflammation and immunity. We previously identified IRF1, IRF3, and IRF4 as regulators of adipogenesis after they emerged from an unbiased search using chromatin state mapping as a tool to identify novel transcription factors $(19,47)$. IRF3 acts as a key downstream transcriptional effector of TLR3 and TLR4 signaling in macrophages; both of these signaling pathways are present in adipocytes (5). Furthermore, the Saltiel group has shown using both genetic and pharmacological approaches that TBK1 and IKK- $\varepsilon$, the upstream activators of IRF3, are activated in the adipose tissue of obese mice and exert negative effects on insulin sensitivity $(21,22)$. These data led us to speculate that IRF3 may act as a transcription factor at the nexus of inflammation and insulin resistance in adipocytes.
In fact, we show that IRF3 levels are elevated in the adipocytes of obese mice and humans, along with known IRF3 target genes. Upstream activation of IRF3 via TLR4 and TLR3 ligation causes insulin resistance in a cell-autonomous manner, and IRF3 is both necessary and sufficient for this effect of TLR signaling. Finally, loss of IRF3 in vivo reduces obesity-induced inflammation and improves insulin sensitivity. Some of this effect could be construed to result from reduced adiposity of $\mathrm{Irf3}^{-/}$mice, but we were able to document improved insulin sensitivity before alterations in body weight developed.

Some of the metabolic effects seen in $\operatorname{Irf} 3^{-1-}$ mice are likely to accrue because of enhanced browning of white fat, specifically in the inguinal depot. This likely accounts for the increased insulin-stimulated glucose uptake seen in subcutaneous fat, as well as the lower body weight of Irf3-\% mice on high-fat diet. Several aspects of the relationship between IRF3 and browning bear special mention. First, the effect of IRF3 on browning is entirely consistent with its role as an innate immune mediator in adipocytes, as TLR4 signaling has recently been shown to suppress adaptive thermogenesis (30). Our data suggest that IRF3 activation may be a mediator of this action of the TLR4 pathway. Second, although several transcription factors have been identified that promote browning, including by our study on IRF4 (17), IRF3 has the opposite effect, acting as a repressor of this activity. The opposing actions of IRF3 and IRF4 on thermogenic genes raise the obvious possibility that they compete for an essential cofactor (such as PGC-1 $\alpha$ ), and/or that they bind competitively to the same genomic loci with different outcomes. To date, we have not identified a clear scenario in which IRF3 and IRF4 exert competing actions, though this is an area of active investigation in the laboratory. Third, the effect may be cell autonomous, as suggested by the fact that isolated SVF from iWAT of $\mathrm{Irf3}^{--}$mice demonstrates enhanced thermogenic gene expression ex vivo. The ability of IRF3 overexpression to reduce thermogenic genes in 3T3-F442A cells also suggests a cell-autonomous effect. Nonetheless, the dramatic induction of M2 polarization in IRF3-deficient animals is at least consistent with the possibility that alternatively activated macrophages may be contributing to the recruitment of beige adipocytes (48). Fourth, while loss of IRF3 has dramatic effects on beige adipocytes, there is no corresponding effect on classic interscapular BAT. This represents an unusual pattern of discrim- 

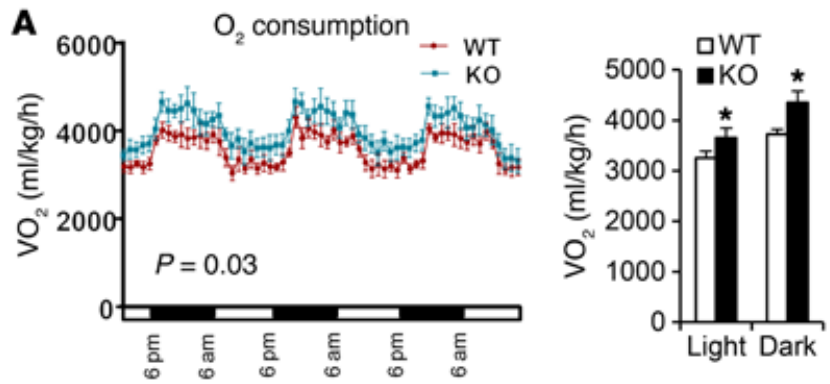
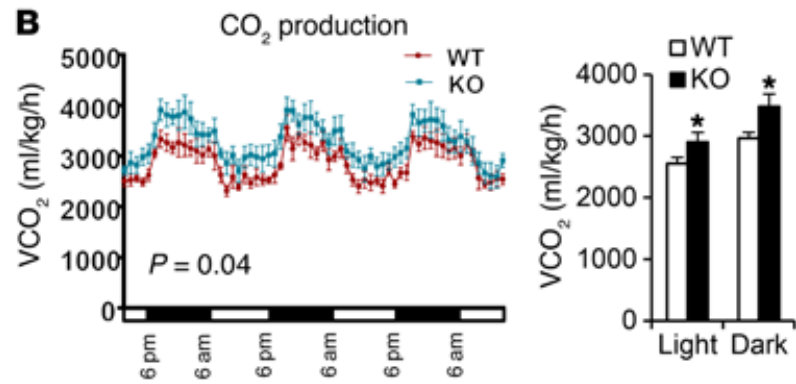

C

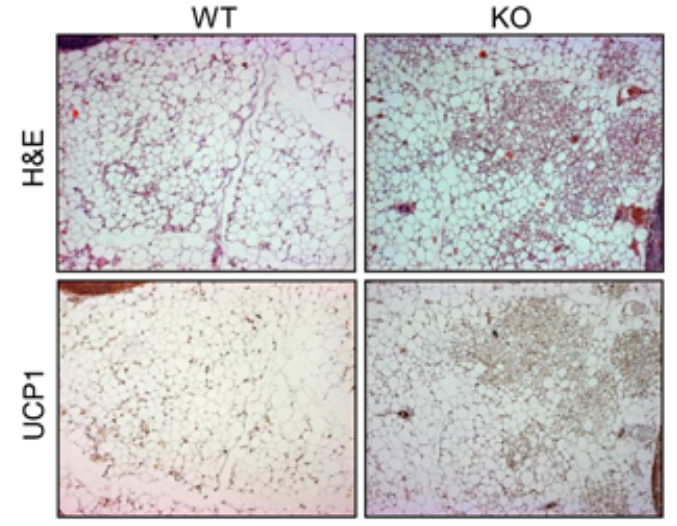

D

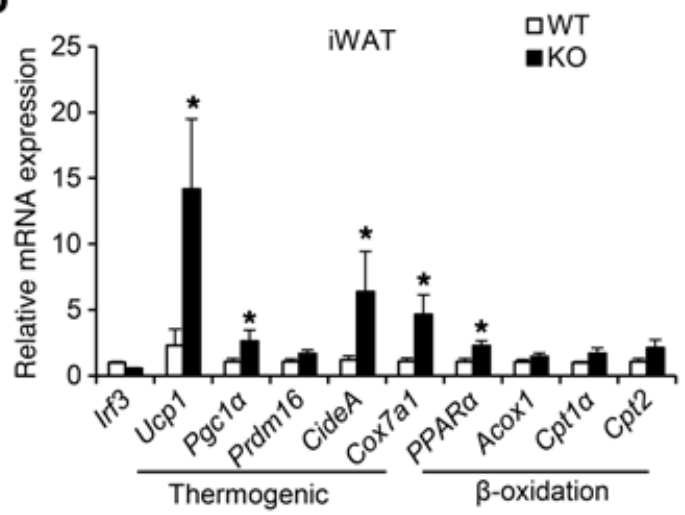

Figure 12. Irf3-/- mice on HFD exhibit increased browning before body weight divergence. Male WT and Irf3 ${ }^{-1-}$ mice were fed HFD and used for CLAMS study. $n=6$ per genotype. (A and B) Oxygen consumption $\left(\mathrm{VO}_{2}\right)(\mathbf{A})$ and carbon dioxide production $\left(\mathrm{VCO}_{2}\right)(\mathbf{B})$ at day and night cycle in WT and Irf3 $3^{-/-}$mice on 8 weeks of HFD. (C) Histology showing H\&E and UCP1 immunostaining in iWAT of WT and Irf3-- mice after 10 weeks of HFD (original magnification, $\times 10)$. (D) Expression of thermogenic and $\beta$-oxidation genes in iWAT of WT and $I r \mathrm{f}^{-/-}$mice on 10 weeks of HFD. Data are presented as mean $\pm \mathrm{SEM}$. For all data, ${ }^{*} P<0.05$.

ination among thermogenic adipocytes, reminiscent of PRDM16, which when knocked out in adipocytes reduces beige fat specifically while leaving brown fat unaffected (49). Given this concordance, we have looked for an interaction between PRDM16 and IRF3, so far without success (not shown). Fifth, and perhaps most interestingly, $\operatorname{Irf3} 3^{-/}$mice show enhanced browning even at thermoneutrality. This indicates that IRF3 acts as a tonic brake on the thermogenic program, such that cold (or another thermogenic stimulus) is no longer required to recruit beige adipocyte formation. The implication is that it may be possible to induce browning and its associated metabolic benefits, without requiring concomitant cold exposure.

In addition to the clear role for beige fat in the metabolic phenotype of $\mathrm{Irf3}^{-/-}$mice, there is likely also a role for WAT, given (a) elevated IRF3 protein levels in fractionated adipocytes from obese mice, (b) selective improvements in insulin-stimulated glucose uptake in WAT, but not in BAT or muscle, (c) specific induction of GLUT4 expression in WAT, but not muscle, and (d) cell-autonomous effects of IRF3 knockdown and overexpression in white adipocytes in vitro. There may also be a role for IRF3 in liver, suggested by reduced liver weight and mild improvement of hepatic gluconeogenesis under basal conditions. Another cell type that expresses IRF3 that could contribute to reduced inflammation and insulin sensitization is the macrophage. $\mathrm{Irf3}^{-/-}$mice show reduced macrophage infiltration into fat pads during a high-fat challenge, and these macrophages display a much higher proportion of alternatively activated versus classically activated cells (i.e., M2 vs. M1). However, it is not clear that macrophages play a major role in the metabolic phenotype of the $\operatorname{Irf} 3^{-/-}$ mice, given that the infiltrating M1 adipose tissue macrophages that emerge in obesity do not express high levels of IRF3 (Figure 1A and Supplemental Figure 1, A-D). Additionally, the cell-autonomous actions of IRF3 in cultured adipocytes suggest that one does not need to invoke a macrophage-based etiology for the phenotype of the knockout mouse. Conditional knockout mice will help us to deconvolute this issue further.

The best-characterized targets of IRF3 in immune cells are inflammatory genes like Ifnb1, Ifna, Ifit1, Ifit2, and Ifit3, but there has never been a comprehensive analysis of IRF3-mediated gene expression in any cell type. Our transcriptional profiling results show that genes from immune-related pathways are among the strongest coordinately regulated genes with overexpression and knockdown of IRF3. These results indicate that IRF3 is a potent driver of the inflammatory response within the adipocyte. One striking finding was the identification of MHC class I genes as targets of IRF3 in adipocytes. It has been reported that during obesity changes in $\mathrm{T}$ cells precede changes in adipose tissue macrophages (50). It was recently proposed that adipocytes can act as antigen-presenting cells via the expression of MHC components, and that this acts as an early trigger in the activation of T cells $(6,26)$. Our results suggest that IRF3 is an early driver of this process, and may thus serve as a critical link between overnutrition and the rest of the well-studied inflammatory cascade in obese adipose tissue. We also identified other nonimmunological IRF3 targets in adipocytes, including Slc2a4 and Adipoq. ChIP sequencing analysis of IRF3 in adipocytes will be required to sort out whether these are direct or indirect targets. 
Interestingly, the $\operatorname{Irf3} 3^{--}$mouse has been reported to exhibit a relatively modest immunological phenotype, with moderately enhanced susceptibility to certain viral infections (28). This is consistent with our data demonstrating a relatively modest metabolic phenotype on chow diet. Once the animal is exposed to high-fat diet, however, the effects of IRF3 ablation become much more apparent. The absence of a strong phenotype until stimulation, either by viral infection or by overnutrition, is predicted based on the fact that IRF3 is cytosolic (and thus sequestered from its DNA targets) until activated. While our own data do not identify the upstream kinases responsible for IRF3 activation in adipocytes, the Saltiel group has used amlexanox, an FDA-approved inhibitor of TBK1 and IKK- $\varepsilon$, to improve metabolic health in ways that are strikingly similar to loss of IRF3 (22).

In summary, we have identified a role of IRF3 as a major transcription factor at the nexus of inflammation and insulin resistance in adipocytes in the state of obesity. Future studies will be required to further elaborate the role of IRF3 and to fully elucidate its tissue-specific target genes and metabolic effects.

\section{Methods}

Reagents. 2-Deoxy-D-[2,6- $\left.{ }^{3} \mathrm{H}\right]$-glucose was purchased from PerkinElmer NEN radiochemicals. Insulin, dexamethasone, isobutylmethylxanthine, LPS, polyinosinic-polycytidylic acid (poly I:C), sodium palmitate, cytochalasin B, glucose, and 2-deoxyglucose were purchased from Sigma-Aldrich.

Antibodies. Antibodies were purchased from Cell Signaling (IRF3, 4302; pIRF3 [S396], 29047; Akt, 9272; pAkt [S473], 9271; p70S6K, 2708; p-p70S6K [T389], 9205; p-p65, 3033; GAPDH, 2118; $\beta$-actin, 4970]), Abcam (F4/80, ab 6640), Santa Cruz Biotechnology Inc. (p65, sc-372; Ikb $\alpha$, sc-371), BioLegend (CD45 [APC/Cy7], 103115; F4/80 [PE/Cy7], 123113; CD11b [Pacific blue], 101223; CD301 [APC], 145707), eBioscience (CD11c [PE], 12-0114-81), and BD Biosciences (caveolin-1, 610059). GLUT4 antibody was a gift from B. Kahn (Beth Israel Deaconess Medical Center, Boston, MA).

Plasmids. The coding sequence of murine IRF3 was amplified by PCR using Phusion DNA polymerase and ligated into the $\mathrm{PCDH}-\mathrm{CMV}$ MCS-EF1-puro lentiviral vector (System Biosciences) at EcoRI and NotI sites. IRF3-2D mutant was generated by site-directed mutagenesis. shIrf3 hairpin designed by the Broad Institute RNAi Consortium (TRCN0000085242) was subcloned into pSIH1-H1-copGFP lentiviral vector (System Biosciences). pSIH construct with the Luciferase shRNA template (shLuc, System Biosciences) was used as control. For adenoviral expression, the PCR product encoding WT murine IRF3 or IRF3-2D mutant was recombined into the pDonR221 plasmid (Invitrogen) using the Gateway technology and was subsequently shuttled into the $\mathrm{pAd} / \mathrm{CMV} / \mathrm{V} 5-\mathrm{DEST}$ (Invitrogen) vector to generate adenoviruses as described below. A $\beta$-galactosidase-V5-encoding adenovirus (Ad-LacZ, Life Technologies) served as a control.

Adenovirus production and purification. Pac1 restriction-digested adenovirus vector pAd/CMV/V5-DEST encoding cDNA for WT murine IRF3 or IRF3-2D mutant was transfected in HEK 293T cells following the manufacturer's instructions, and crude adenovirus was amplified twice. Large-scale production of high-titer adenovirus was performed using Vivapure AdenoPACK 100 purification kit (Sartorius Stedim Biotech). Purified adenovirus titer was determined using Adeno-X-Rapit Titer kit (Clontech). For differentiated F442A adipocytes, LacZ, IRF3, or IRF3-2D adenovirus $\left(1 \times 10^{11}\right.$ infectious units $\left./ \mathrm{ml}\right)$ was used.
Cell culture. 3T3-L1 preadipocytes (American Type Culture Collection) were cultured in DMEM (Invitrogen) with 10\% bovine calf serum (HyClone) at $37^{\circ} \mathrm{C}$ and $5 \% \mathrm{CO}_{2}$. Two-days-postconfluent cells were treated with DMEM containing 10\% FBS (Invitrogen), $5 \mu \mathrm{g} / \mathrm{ml}$ insulin, $0.5 \mathrm{mM}$ isobutylmethylxanthine, and $1 \mu \mathrm{M}$ dexamethasone for 48 hours. Cells were maintained in culture medium containing FBS and insulin for another 2 days. Thereafter, cells were maintained in culture medium containing FBS only. Lentivirus particles were generated by cotransfection of lentiviral constructs with pM2DG and PsPAX2 plasmids in HEK 293T cells. Lentivirus-containing supernatant was collected after 48 hours of transduction, filtered through 0.45 $\mu \mathrm{M}$ filter, and added onto mature 3T3-L1 adipocytes with $0.8 \mu \mathrm{g} / \mathrm{ml}$ Polybrene (Sigma-Aldrich) for 24 hours. 3T3-L1 adipocytes were used for experiments after 6 days of lentivirus transduction. Transduction efficiency was determined by comparison with cells transduced in parallel with EGFP lentivirus. 3T3-L1 adipocytes were differentiated fully for 8 days, treated with lentiviral supernatant for another 6 days, and then treated with various doses of LPS for 6 days prior to glucose uptake assays, or harvested for RNA or protein. Similarly, mature 3T3L1 adipocytes were treated with various doses of palmitate conjugated to BSA (3:1 ratio) for 48 hours or poly I:C for 24 hours before glucose uptake assays or harvested for protein.

3T3-F442A preadipocytes (gift from P. Puigserver, Dana-Farber Cancer Institute, Boston, MA) were differentiated in DMEM containing $10 \% \mathrm{FBS}, 5 \mu \mathrm{g} / \mathrm{ml}$ insulin, and $1 \mu \mathrm{M}$ rosiglitazone for 48 hours. Cells were maintained in DMEM containing $5 \mu \mathrm{g} / \mathrm{ml}$ insulin for another 2 days. Thereafter, cells were maintained in medium containing FBS only and used for experiments on day 6.

Glucose uptake in 3T3-L1 adipocytes. Glucose uptake assays were performed as described earlier (39). Briefly, mature 3T3-L1 adipocytes were incubated in serum-free DMEM for 4-6 hours. Cells were then washed 3 times with KRH buffer (12 mM Hepes, pH 7.4, $121 \mathrm{mM} \mathrm{NaCl}, 5 \mathrm{mM}$ $\mathrm{KCl}, 0.33 \mathrm{mM} \mathrm{CaCl}_{2}$, and $1.2 \mathrm{mM} \mathrm{MgSO}_{4}$ ) and incubated for $20 \mathrm{~min}$ utes in KRH buffer in the absence or presence of $100 \mathrm{nM}$ insulin. Cells were treated with 2 -deoxy-D-[2,6- $\left.{ }^{3} \mathrm{H}\right]$-glucose $(0.33 \mu \mathrm{Ci} / \mathrm{ml})$ for another 10 minutes. Glucose uptake was stopped quickly by 3 rapid washes with KRH buffer containing $200 \mathrm{mM}$ glucose and $10 \mu \mathrm{M}$ cytochalasin B on ice. Cells were solubilized in $0.1 \%$ SDS for 30 minutes, and radioactivity was measured by liquid scintillation counting. Total protein was determined by BCA method (Pierce), and results were normalized to protein amount.

$N F-\kappa B$ activity. Nuclear fractions from 3T3-L1 adipocytes were isolated using Nuclear Extraction Kit (Active Motif) per the manufacturer's protocol. Ten micrograms of isolated nuclear extract protein

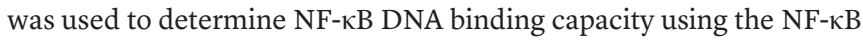
TransAm Kit (Active Motif).

$R N A$ isolation and gene expression analysis by qPCR. Total RNA was extracted from cells or tissues using TRIzol reagent (Invitrogen) following the manufacturer's instructions. Fat pads were fractionated into adipocytes and stromal-vascular cells as described in ref. 19. One microgram of total RNA was reverse-transcribed to first-strand cDNA using RETROscript First Strand Synthesis Kit (Ambion) with oligo-dT primers. Quantitative PCR (qPCR) was performed using synthesized cDNA with SYBR Green qPCR Master Mix (Applied Biosystems) and specific primers using a 7900HT Fast Real Time PCR System (Applied Biosystems). Primer sequences are listed in Supplemental Table 6. The relative abundance of mRNA was normalized to 36B4 mRNA as the invariant control using the $\Delta \Delta \mathrm{Ct}$ method (where $\mathrm{Ct}$ is the threshold cycle) (51). 
RNA-seq. mRNA was purified from 70 ng of total RNA using the Ribo-Zero Magnetic Gold Kit (catalog MRZG126, Illumina). Libraries were prepared using the TruSeq RNA Library Preparation Kit v2 (catalog RS-122-2001, Illumina) according to the manufacturer's protocol starting with the EPF step. Sequencing was performed on the Illumina HiSeq2500. RNA-seq data are aligned using TopHat2 (52). Reads are assigned to transcripts using featureCounts and an $\mathrm{mm} 9$ genome modified to minimize overlapping transcripts (53). Differential expression analysis of the data is performed using EdgeR (54). Gene set enrichment analysis is carried out with RDAVIDWebService (55) and GOstats (56) against the GO and KEGG (Kyoto Encyclopedia of Genes and Genomes) databases.

Accession number. The RNA-seq data set generated for this paper can be accessed at Gene Expression Omnibus (GEO) (accession number GSE81193).

Animals. Irf $3^{-/-}$mice were obtained from RIKEN BRC Experimental Animal Division (28). Experimental cohorts were generated by breeding of $\operatorname{Irf} 3^{+/-}$males to $\operatorname{Irf} 3^{+/}$females. Mice were maintained on a standard chow diet (8664 Harlan Teklad, 6.4\% wt/wt fat) under a regular 12 hours light/12 hours dark cycle at constant temperature $\left(23^{\circ} \mathrm{C}\right)$. High-fat diet experiments in male $\mathrm{Irf3}^{-/-}$mice were performed by feeding of high-fat diet (12331i, Research Diets Inc.) to 6-week-old mice for 10-16 weeks. Body weight was measured weekly. At the end of experiments, tissues were collected, flash-frozen in liquid nitrogen, and stored at $-80^{\circ} \mathrm{C}$ until use. Mouse body mass composition was examined by MRI (Echo Medical Systems).

Energy expenditure measurement. Metabolic rate was measured by indirect calorimetry in open-circuit Oxymax chambers that are a component of the Comprehensive Lab Animal Monitoring System (CLAMS, Columbus Instruments). All mice were acclimatized to monitoring cages for 48 hours prior to the beginning of an additional 72 hours of hourly automated recordings of physiological parameters. Mice were housed singly and maintained under a 12 hours light/12 hours dark cycle at $23^{\circ} \mathrm{C}$. Food and water were available ad libitum. Data were analyzed using 2-way ANOVA.

Glucose and insulin tolerance tests. To determine glucose tolerance, mice were fasted for 12 hours and glucose ( $1 \mathrm{~g} / \mathrm{kg}$ body weight) was administered i.p. Blood samples were collected at 0, 15, 30, 60, 90, and 120 minutes. Blood glucose levels were measured with a portable glucometer. To determine insulin tolerance, mice were fasted for 6 hours and insulin $(0.8 \mathrm{U} / \mathrm{kg}$ body weight) was administered i.p. Blood glucose levels were determined at 0, 15, 30, 60, 90, and 120 minutes.

Analysis of plasma parameters. Blood samples were collected from fed (ad libitum) or fasted (14-hour food withdrawal) animals in EDTA-coated blood collection tubes, and plasma insulin levels were quantified using an ELISA kit from Crystal Chem following the manufacturer's instructions. Plasma levels of triglycerides and FFAs were determined using commercially available kits (Thermo Scientific and Abcam, respectively).

Insulin signaling. Mice were fasted overnight for insulin signaling studies, and insulin (10 U/kg body weight) was administered i.p. After 10 minutes various tissues were harvested and stored at $-80^{\circ} \mathrm{C}$ until use. Tissue samples were homogenized in cell signaling lysis buffer containing protease inhibitors (Roche) and phosphatase inhibitors (Sigma-Aldrich) and subjected to Western blotting.

Measurement of cellular respiration. Tissue respiration was determined using a Clark electrode (Strathkelvin Instruments). Freshly dissected iWAT and eWAT were rinsed in sterile saline, weighed, minced, and placed into the respiration buffer (PBS, 2\% BSA, $25 \mathrm{mM}$ glucose, and $1 \mathrm{mM}$ pyruvate). For each tissue, readings were taken in triplicate using 3 separate pieces of tissue of equivalent size. Oxygen consumption rate was normalized to tissue weight.

Isolation and differentiation of cells from SVF. Dissected iWAT from 6- to 8-week-old WT and Irf3 ${ }^{-1-}$ mice was digested in buffer (collagenase $\mathrm{D}[2.5 \mathrm{U} / \mathrm{ml}]$, dispase II [2.4 U/ml], $\mathrm{CaCl}_{2}$ [10 mM] in PBS) for 30 minutes at $37^{\circ} \mathrm{C}$ in a prewarmed water bath. Digestion was neutralized with culture media (complete DMEM/F12 [1:1] containing GlutaMAX [Thermo Fisher], 10\% FBS, and Penicillin Streptomycin [Thermo Fisher]) and filtered through a $100-\mu \mathrm{m}$ cell strainer. The suspension was centrifuged at $500 \mathrm{~g}$ for 5 minutes at $4^{\circ} \mathrm{C}$. Floating adipocytes and lipid layer were aspirated; the cell pellet was resuspended in culture media, filtered through a $40-\mu \mathrm{m}$ cell strainer, and centrifuged as described above. The pellet containing cells from the stromal-vascular fraction (SVF) was seeded in a $10-\mathrm{cm}$ dish until 50\% confluence. Two-days-postconfluent SVF cells seeded in a 12-well plate were differentiated with $5 \mu \mathrm{g} / \mathrm{ml}$ insulin, $0.5 \mathrm{mM}$ isobutylmethylxanthine, 1 $\mu \mathrm{M}$ dexamethasone, and $1 \mu \mathrm{M}$ rosiglitazone for 48 hours. Cells were maintained in culture media containing $5 \mu \mathrm{g} / \mathrm{ml}$ insulin until day 6 and harvested for RNA at day 10.

FACS and analysis. Epididymal and inguinal fat pads from chowor high-fat-diet mice were digested in collagenase $(1 \mathrm{mg} / \mathrm{ml})$ for 30 minutes at $37^{\circ} \mathrm{C}$. Digested cells were filtered through a $100-\mu \mathrm{m}$ cell strainer and centrifuged at $500 \mathrm{~g}$ for 10 minutes at $4^{\circ} \mathrm{C}$. Isolated SVFs were erythrocyte depleted with rbc lysis buffer and filtered through a $40-\mu \mathrm{m}$ cell strainer. SVFs were resuspended in FACS buffer (PBS containing 2\% FBS) and stained with the indicated fluorescence-labeled antibodies or isotype control for 30 minutes at $4^{\circ} \mathrm{C}$ followed by washing with FACS buffer. To analyze M1 and M2 macrophages, SVFs were stained with CD11c (also known as integrin alpha X, Itgax) and CD301 (also known as macrophage galactose-type calcium-type lectin, $\mathrm{Mgl}$ ) antibody, respectively (3). For macrophage analysis, cells were stained with Zombie Aqua Fixable Viability dye (Biolegend), and live-gated cells were sorted on a BD FACSAria II flow cytometer (BD Biosciences) at the Beth Israel Deaconess Medical Center Flow Cytometry Core. Cells were stained with DAPI for gating live cells to sort macrophages. Data were analyzed using Kaluza software (Beckman Coulter).

Tissue histology, IHC, and confocal staining. Fat tissues collected from mice were immediately fixed in $4 \%$ formaldehyde, incubated overnight at $4^{\circ} \mathrm{C}$, and washed with $70 \%$ ethanol. Formalin-fixed paraffin-embedded tissues were stained with $\mathrm{H} \& \mathrm{E}$ or processed for IHC or confocal imaging. For IHC, sections of formalin-fixed paraffin-embedded fat tissues were incubated with anti-F4/80 antibody (ab 6640, 1:500) overnight at room temperature. After washing, the bound antibody was detected with a labeled polymer-HRP (Envision Plus kit) using DAB chromogen. High-resolution digital images were obtained using the 3DHistech Panoramic Scanner. For confocal imaging, eWAT and iWAT tissue sections were incubated with antiF4/80 antibody (1:200) overnight at $4^{\circ} \mathrm{C}$, washed, and incubated with appropriate secondary antibody conjugated with Alexa Fluor 647 (ab 150115). Samples were counterstained with DAPI (Vectashield, Vector Laboratories). Fluorescent images were taken on a laser-scanning Zeiss LSM 710 inverted confocal microscope.

Quantification of plasma cytokines and chemokines. Plasma cytokines and chemokines were measured using MILLIPLEX MAP Mouse Cytokine/Chemokine Magnetic Bead Panel kit (catalog MCYT- 
MAG-70K-PX32, EMD Millipore Corp.) following the manufacturer's instructions. Data from the reactions were acquired using the Luminex MILLIPLEX analyzer, while a digital processor managed data output, and the MILLIPLEX analyst software was used to determine fluorescence intensity (MFI) and concentration (pg/ml).

Western blotting. Cell lysates (10 $\mu \mathrm{g}$ protein), cell fractions (10-50 $\mu \mathrm{g}$ protein), or tissue lysates $(20-50 \mu \mathrm{g}$ protein) were separated by 4\%-15\% gradient SDS-PAGE and transferred to nitrocellulose membrane (Bio-Rad). Blots were incubated with appropriate primary and HRP-conjugated secondary antibodies. Detection was performed with ECL (Western Lightning Plus-ECL, PerkinElmer Inc.) and exposed to X-ray Film (Phoenix Research Products). All blots were quantified using Image J software (NIH, Bethesda, MD). To see unedited gels and blots, please see Supplemental Material.

Human studies. A total of 48 nondiabetic and 45 type 2 diabetic individuals were recruited in the study. The clinicodemographic data of the study participants are summarized in Supplemental Table 1. Anthropometric and clinical measurements were taken by measuring of height and weight using calibrated portable electronic weighing scales and portable inflexible height measuring bars. BMI was calculated using the standard BMI formula, body weight $(\mathrm{kg}) / \mathrm{height} \mathrm{t}^{2}\left(\mathrm{~m}^{2}\right)$. Peripheral blood was collected from overnight-fasted individuals and analyzed for fasting glucose, glycated hemoglobin $\left(\mathrm{HbA}_{1 \mathrm{c}}\right)$, fasting insulin, and lipid profile using standard clinical laboratory procedures.

Collection of human subcutaneous adipose tissue samples and realtime $q R T-P C R$. Human adipose tissue samples ( 0.5 g) were collected via abdominal subcutaneous fat pad biopsy lateral to the umbilicus using standard surgical method. Freshly collected adipose tissue samples $(\sim 50-100 \mathrm{mg})$ were preserved in RNAlater stored at $-80^{\circ} \mathrm{C}$ until use. Total cellular RNA was purified using RNeasy kit (Qiagen) per the manufacturer's instructions. RNA samples (1 $\mu \mathrm{g}$ each) were reverse transcribed to yield cDNA using random hexamer primers and TaqMan reverse transcription reagents (High Capacity cDNA Reverse Transcription kit, Applied Biosystems). For real-time qRTPCR, cDNA (50 ng) was amplified using TaqMan Gene Expression MasterMix (Applied Biosystems) and gene-specific 20× TaqMan Gene Expression Assays for IRF3 (Hs01547283_m1) and GAPDH (Hs03929097_g1) (Applied Biosystems) containing forward and reverse primers and a target-specific TaqMan minor groove binder (MGB) probe labeled with 6-fluorescein amidite dye at the $5^{\prime}$ end and nonfluorescent quencher-MGB at the $3^{\prime}$ end of the probe, for 40 cycles of PCR reaction using a 7500 Fast Real-Time PCR System (Applied Biosystems). Each cycle consisted of denaturation for $15 \mathrm{sec}-$ onds at $95^{\circ} \mathrm{C}$ and annealing/extension for 1 minute at $60^{\circ} \mathrm{C}$, which started after uracil DNA glycosylase activation $\left(50^{\circ} \mathrm{C}\right.$ for 2 minutes) and AmpliTaq Gold enzyme activation $\left(95^{\circ} \mathrm{C}\right.$ for 10 minutes). The amplified GAPDH expression was used as internal control to normalize the differences in individual samples, and gene expression level of IRF3 relative to controls (lean adipose tissue) was calculated using the $-2^{\Delta \Delta \mathrm{Ct}}$ method. Relative mRNA expression was measured as fold expression over average of control gene expression. The expression level in control samples was assumed as 1 , and data were presented as mean \pm SEM values.

Hyperinsulinemic-euglycemic clamp. All procedures required for the hyperinsulinemic-euglycemic clamp were approved by the Vanderbilt University Animal Care and Use Committee. Catheters were implanted into a carotid artery and a jugular vein of mice for sampling and infusions, respectively, 5 days before the study, as described by Berglund et al. (57). Insulin clamps were performed on mice fasted for 5 hours using a modification of the method described by Ayala et al. $(58) \cdot\left[3-{ }^{3} \mathrm{H}\right]$-glucose was primed $(1.5 \mu \mathrm{Ci})$ and continuously infused for a 90 -minute equilibration and basal sampling periods $(0.075 \mu \mathrm{Ci} /$ min). $\left[3-{ }^{3} \mathrm{H}\right]$-glucose was mixed with the nonradioactive glucose infusate (infusate-specific activity of $0.5 \mu \mathrm{Ci} / \mathrm{mg}$ ) during the 2-hour clamp period. Arterial glucose was clamped using a variable rate of glucose (plus trace $\left[3{ }^{-3} \mathrm{H}\right]$-glucose) infusion, which was adjusted on the basis of the measurement of blood glucose at 10-minute intervals. By mixing of radioactive glucose with the nonradioactive glucose infused during a clamp, deviations in arterial glucose-specific activity are minimized and steady-state conditions are achieved. The calculation of glucose kinetics is therefore more robust (59). Baseline blood or plasma variables were calculated as the mean of values obtained in blood samples collected at -15 and -5 minutes. At time zero, insulin infusion ( $2.5 \mathrm{mU} / \mathrm{kg}$ of body weight per minute) was started and continued for 120 minutes. Mice received heparinized saline-washed erythrocytes from donors at $5 \mu \mathrm{l} / \mathrm{min}$ to prevent a fall in hematocrit. Blood was taken from 80 to 120 minutes for the determination of $\left[3-{ }^{3} \mathrm{H}\right]$-glucose. Clamp insulin was determined at $t=100$ and 120 minutes. At 120 minutes, $13 \mu \mathrm{Ci}$ of $2-\left[{ }^{14} \mathrm{C}\right]$-deoxyglucose $\left(\left[{ }^{14} \mathrm{C}\right]-2 \mathrm{DG}\right)$ was administered as an i.v. bolus. Blood was taken from 2 to $25 \mathrm{~min}-$ utes for determination of $\left[{ }^{14} \mathrm{C}\right]-2 \mathrm{DG}$. After the last sample, mice were anesthetized and tissues were freeze-clamped for biochemical analysis. Plasma insulin was determined by RIA. Radioactivity of $\left[3-{ }^{3} \mathrm{H}\right]$-glucose and $\left[{ }^{14} \mathrm{C}\right]-2 \mathrm{DG}$ in plasma samples, and $\left[{ }^{14} \mathrm{C}\right]-2 \mathrm{DG}-6-$ phosphate in tissue samples, was determined by liquid scintillation counting. Glucose appearance ( $\mathrm{Ra}$ ) and disappearance ( $\mathrm{Rd}$ ) rates were determined using steady-state equations (60). Endogenous glucose appearance (endoRa) was determined by subtraction of the glucose infusion rate from total Ra. The glucose metabolic index (Rg) was calculated as previously described (61).

Statistics. Biochemical and physiological data are presented as mean \pm SEM. Statistical significance was measured by Student's unpaired $t$ tests (2-tailed). Energy expenditure statistical analysis was performed using 2-way ANOVA for repeated measures followed by post hoc Bonferroni analysis. RNA-seq studies were analyzed as described above. A $P$ value less than 0.05 was considered significant.

Study approval. All animal studies were approved by the Beth Israel Deaconess Medical Center IACUC and were in accordance with NIH guidelines. The human studies were approved by the ethics committee of Dasman Diabetes Institute, Kuwait. All participants gave written informed consent.

Supplemental data. Supplemental information (online) includes 10 figures and 6 tables.

\section{Author contributions}

MK, XW, JE, and EDR designed the experiments. Experiments were carried out by MK and XW with help from HCR, XK, and LK. RNA-seq was performed by MK and DT. Computational analysis was done by AL. SK performed glucose uptake with LPS treatment. LL performed the hyperinsulinemic-euglycemic clamp study. RA supervised experiments with human samples and confocal imaging of mouse fat tissues on high-fat diet, as well as some immunological measurements. EDR supervised all experiments. MK and EDR analyzed all the data and wrote the manuscript. 


\section{Acknowledgments}

This work was funded by NIH R01 DK085171 and DK102170 to E.D. Rosen, and by funds from the Dasman Diabetes Institute and the Kuwait Foundation for the Advancement of Sciences. We thank Ines Sousa Lima for assistance in insulin signaling studies. Eleanna DeFilippis, Linus Tsai, and $\mathrm{Su} \mathrm{Xu}$ provided technical support and helpful discussions. Valerie Atizado and Puthiyaveetil Shihab helped with IHC and qRT-PCR of human samples, respectively. We also thank the Vanderbilt Mouse
Metabolic Phenotyping Center (DK059637) for performing the hyperinsulinemic-euglycemic clamps. The Vanderbilt University Hormone Assay and Analytical Core performed the hormone analysis (DK059637 and DK020593).

Address correspondence to: Evan D. Rosen, Beth Israel Deaconess Medical Center, Harvard Medical School, 330 Brookline Avenue, E/CLS743, Boston, Massachusetts 02215, USA. Phone: 617.735.3221; E-mail: erosen@bidmc.harvard.edu.
1. Osborn O, Olefsky JM. The cellular and signaling networks linking the immune system and metabolism in disease. Nat Med. 2012;18(3):363-374.

2. Odegaard JI, Chawla A. The immune system as a sensor of the metabolic state. Immunity. 2013;38(4):644-654.

3. Lumeng CN, Bodzin JL, Saltiel AR. Obesity induces a phenotypic switch in adipose tissue macrophage polarization. JClin Invest. 2007;117(1):175-184.

4. Mathis D. Immunological goings-on in visceral adipose tissue. Cell Metab. 2013;17(6):851-859.

5. Jialal I, Kaur H, Devaraj S. Toll-like receptor status in obesity and metabolic syndrome: a translational perspective. JClin Endocrinol Metab. 2014;99(1):39-48.

6. Deng T, et al. Class II major histocompatibility complex plays an essential role in obesity-induced adipose inflammation. Cell Metab. 2013;17(3):411-422.

7. Kim F, et al. Toll-like receptor- 4 mediates vascular inflammation and insulin resistance in dietinduced obesity. Circ Res. 2007;100(11):1589-1596.

8. Pierre N, Deldicque L, Barbé C, Naslain D, Cani PD, Francaux M. Toll-like receptor 4 knockout mice are protected against endoplasmic reticulum stress induced by a high-fat diet. PLoS One. 2013;8(5):e65061.

9. Shi H, Kokoeva MV, Inouye K, Tzameli I, Yin H, Flier JS. TLR4 links innate immunity and fatty acid-induced insulin resistance. J Clin Invest. 2006;116(11):3015-3025.

10. Vallabhapurapu S, Karin M. Regulation and function of NF- $\mathrm{\kappa B}$ transcription factors in the immune system. Annu Rev Immunol. 2009;27:693-733.

11. Berg AH, Lin Y, Lisanti MP, Scherer PE. Adipocyte differentiation induces dynamic changes in NF- $\mathrm{KB}$ expression and activity. Am J Physiol Endocrinol Metab. 2004;287(6):E1178-E1188.

12. Cai D, et al. Local and systemic insulin resistance resulting from hepatic activation of IKK- $\beta$ and NF-кB. Nat Med. 2005;11(2):183-190.

13. Gao Z, et al. P65 inactivation in adipocytes and macrophages attenuates adipose inflammatory response in lean but not in obese mice. Am JPhysiol Endocrinol Metab. 2015;308(6):E496-E505.

14. Hinz M, Scheidereit C. The I $\mathrm{B}$ kinase complex in NF- $\mathrm{KB}$ regulation and beyond. EMBO Rep. 2014;15(1):46-61.

15. Tang $\mathrm{T}$, et al. Uncoupling of inflammation and insulin resistance by NF- $\mathrm{kB}$ in transgenic mice through elevated energy expenditure. J Biol Chem. 2010;285(7):4637-4644.

16. Honda K, Taniguchi T. IRFs: master regulators of signalling by Toll-like receptors and cytosolic pattern-recognition receptors. Nat Rev Immunol. 2006;6(9):644-658.

17. Kong X, et al. IRF4 is a key thermogenic transcriptional partner of PGC-1 $\alpha$. Cell. 2014;158(1):69-83.

18. Eguchi J, et al. Transcriptional control of adipose lipid handling by IRF4. Cell Metab. 2011;13(3):249-259.

19. Eguchi J, et al. Interferon regulatory factors are transcriptional regulators of adipogenesis. Cell Metab. 2008;7(1):86-94.

20. Fitzgerald KA, et al. IKKepsilon and TBK1 are essential components of the IRF3 signaling pathway. Nat Immunol. 2003;4(5):491-496.

21. Chiang SH, et al. The protein kinase IKK $\varepsilon$ regulates energy balance in obese mice. Cell. 2009;138(5):961-975.

22. Reilly SM, et al. An inhibitor of the protein kinases TBK1 and IKK- $\varepsilon$ improves obesity-related metabolic dysfunctions in mice. Nat Med. 2013;19(3):313-321.

23. Lin R, Heylbroeck C, Pitha PM, Hiscott J. Virusdependent phosphorylation of the IRF-3 transcription factor regulates nuclear translocation, transactivation potential, and proteasome-mediated degradation. Mol Cell Biol. 1998;18(5):2986-2996.

24. Doyle S, et al. IRF3 mediates a TLR3/TLR4specific antiviral gene program. Immunity. 2002;17(3):251-263.

25. Chen W, Srinath H, Lam SS, Schiffer CA, Royer WE, Lin K. Contribution of Ser386 and Ser396 to activation of interferon regulatory factor 3. J Mol Biol. 2008;379(2):251-260.

26. Xiao L, et al. Large adipocytes function as antigen-presenting cells to activate $\mathrm{CD} 4(+) \mathrm{T}$ cells via upregulating MHCII in obesity. Int JObes (Lond). 2016;40(1):112-120.

27. Kang S, et al. Identification of nuclear hormone receptor pathways causing insulin resistance by transcriptional and epigenomic analysis. Nat Cell Biol. 2015;17(1):44-56.

28. Sato M, et al. Distinct and essential roles of transcription factors IRF-3 and IRF-7 in response to viruses for IFN $\alpha / \beta$ gene induction. Immunity. 2000;13(4):539-548.

29. Carvalho E, Jansson PA, Nagaev I, Wenthzel AM, Smith U. Insulin resistance with low cellular IRS-1 expression is also associated with low GLUT4 expression and impaired insulin-stimulated glucose transport. FASEB J. 2001;15(6):1101-1103.

30. Okla M, Wang W, Kang I, Pashaj A, Carr T, Chung S. Activation of Toll-like receptor 4 (TLR4) attenuates adaptive thermogenesis via endoplasmic reticulum stress. J Biol Chem.
2015;290(44):26476-26490.

31. Harms M, Seale P. Brown and beige fat: development, function and therapeutic potential. Nat Med. 2013;19(10):1252-1263.

32. Odegaard JI, Chawla A. Pleiotropic actions of insulin resistance and inflammation in metabolic homeostasis. Science. 2013;339(6116):172-177.

33. Olefsky JM, Glass CK. Macrophages, inflammation, and insulin resistance. Аnпи Rev Physiol. 2010;72:219-246.

34. Ye J. Emerging role of adipose tissue hypoxia in obesity and insulin resistance. Int JObes (Lond). 2009;33(1):54-66.

35. Halberg $\mathrm{N}$, et al. Hypoxia-inducible factor $1 \alpha$ induces fibrosis and insulin resistance in white adipose tissue. Mol Cell Biol. 2009;29(16):4467-4483.

36. Sun K, Tordjman J, Clement K, Scherer PE. Fibrosis and adipose tissue dysfunction. Cell Metab. 2013;18(4):470-477.

37. Hotamisligil GS. Inflammation and endoplasmic reticulum stress in obesity and diabetes. Int $J$ Obes (Lond). 2008;32(suppl 7):S52-S54.

38. Ozcan U, et al. Endoplasmic reticulum stress links obesity, insulin action, and type 2 diabetes. Science. 2004;306(5695):457-461.

39. Houstis N, Rosen ED, Lander ES. Reactive oxygen species have a causal role in multiple forms of insulin resistance. Nature. 2006;440(7086):944-948.

40. Henriksen EJ, Diamond-Stanic MK, Marchionne EM. Oxidative stress and the etiology of insulin resistance and type 2 diabetes. Free Radic Biol Med. 2011;51(5):993-999.

41. Cani PD, et al. Metabolic endotoxemia initiates obesity and insulin resistance. Diabetes. 2007;56(7):1761-1772.

42. Creely SJ, et al. Lipopolysaccharide activates an innate immune system response in human adipose tissue in obesity and type 2 diabetes. $A m J$ Physiol Endocrinol Metab. 2007;292(3):E740-E747.

43. Opie LH, Walfish PG. Plasma free fatty acid concentrations in obesity. N Engl JMed. 1963;268:757-760.

44. Freaney JE, Kim R, Mandhana R, Horvath CM. Extensive cooperation of immune master regulators IRF3 and NFKB in RNA Pol II recruitment and pause release in human innate antiviral transcription. Cell Rep. 2013;4(5):959-973.

45. Wietek C, Miggin SM, Jefferies CA, O'Neill LA. Interferon regulatory factor-3-mediated activation of the interferon-sensitive response element by Toll-like receptor (TLR) 4 but not TLR3 requires the p65 subunit of NF-к. J Biol Chem. 2003;278(51):50923-50931.

46. Zhang X, Zhang G, Zhang H, Karin M, Bai H, Cai 
D. Hypothalamic IKK $\beta / N F-\kappa B$ and ER stress link overnutrition to energy imbalance and obesity. Cell. 2008;135(1):61-73.

47. Mikkelsen TS, et al. Comparative epigenomic analysis of murine and human adipogenesis. Cell. 2010;143(1):156-169.

48. Nguyen KD, et al. Alternatively activated macrophages produce catecholamines to sustain adaptive thermogenesis. Nature. 2011;480(7375):104-108.

49. Cohen P, et al. Ablation of PRDM16 and beige adipose causes metabolic dysfunction and a subcutaneous to visceral fat switch. Cell. 2014;156(1-2):304-316.

50. Nishimura S, et al. CD8 $8^{+}$effector T cells contribute to macrophage recruitment and adipose tissue inflammation in obesity. Nat Med. 2009;15(8):914-920.

51. Livak KJ, Schmittgen TD. Analysis of relative gene expression data using real-time quantitative PCR and the $2(-\Delta \Delta \mathrm{C}(\mathrm{T}))$ Method. Methods.
2001;25(4):402-408.

52. Kim D, Pertea G, Trapnell C, Pimentel H, Kelley R, Salzberg SL. TopHat2: accurate alignment of transcriptomes in the presence of insertions, deletions and gene fusions. Genome Biol. 2013;14(4):R36.

53. Liao Y, Smyth GK, Shi W. featureCounts: an efficient general purpose program for assigning sequence reads to genomic features. Bioinformatics. 2014;30(7):923-930.

54. Robinson MD, McCarthy DJ, Smyth GK. edgeR: a Bioconductor package for differential expression analysis of digital gene expression data. Bioinformatics. 2010;26(1):139-140.

55. Fresno C, Fernández EA. RDAVIDWebService: a versatile R interface to DAVID. Bioinformatics. 2013;29(21):2810-2811.

56. Falcon S, Gentleman R. Using GOstats to test gene lists for GO term association. Bioinformatics. 2007;23(2):257-258.
57. Berglund ED, et al. Glucose metabolism in vivo in four commonly used inbred mouse strains. Diabetes. 2008;57(7):1790-1799.

58. Ayala JE, Bracy DP, McGuinness OP, Wasserman DH. Considerations in the design of hyperinsulinemic-euglycemic clamps in the conscious mouse. Diabetes. 2006;55(2):390-397.

59. Finegood DT, Bergman RN, Vranic M. Estimation of endogenous glucose production during hyperinsulinemic-euglycemic glucose clamps. Comparison of unlabeled and labeled exogenous glucose infusates. Diabetes. 1987;36(8):914-924.

60. Steele R, Wall JS, De Bodo RC, Altszuler N. Measurement of size and turnover rate of body glucose pool by the isotope dilution method. Am J Physiol. 1956;187(1):15-24.

61. Kraegen EW, James DE, Jenkins AB, Chisholm DJ. Dose-response curves for in vivo insulin sensitivity in individual tissues in rats. Am J Physiol. 1985;248(3 pt 1):E353-E362. 